\title{
Optimal Design of Submarine Pipelines by a Genetic Algorithm with Embedded On-Bottom Stability Criteria
}

\author{
Juliana Souza Baioco $\left(D,{ }^{1,2}\right.$ Mauro Henrique Alves de Lima Jr., \\ Carl Horst Albrecht $\mathbb{D}^{1},{ }^{1}$ Beatriz Souza Leite Pires de Lima $\left(\mathbb{D},{ }^{1}\right.$ \\ Breno Pinheiro Jacob ${ }^{(D)}{ }^{1}$ and Djalene Maria Rocha ${ }^{3}{ }^{3}$
}

\begin{abstract}
${ }^{1}$ Laboratory of Computer Methods and Offshore Systems (LAMCSO), PEC/COPPE/UFRJ, Civil Engineering Department, Post-Graduate Institute of the Federal University of Rio de Janeiro, Avenida Pedro Calmon, S/N, Cidade Universitária, Ilha do Fundão, 21941-596 Rio de Janeiro, RJ, Brazil

${ }^{2}$ Chemical and Petroleum Engineering Department (TEQ), UFF, Rua Passo da Pátria No. 156, São Domingos, 24210-240 Niterói, RJ, Brazil

${ }^{3}$ Petróleo Brasileiro S.A. (Petrobras), Research \& Development Center (CENPES), Avenida Horacio Macedo 950, Cidade Universitária, Ilha do Fundão, 21941-915 Rio de Janeiro, RJ, Brazil
\end{abstract}

Correspondence should be addressed to Breno Pinheiro Jacob; breno@lamcso.coppe.ufrj.br

Received 27 July 2017; Accepted 22 January 2018; Published 11 March 2018

Academic Editor: Maurizio Brocchini

Copyright ( 2018 Juliana Souza Baioco et al. This is an open access article distributed under the Creative Commons Attribution License, which permits unrestricted use, distribution, and reproduction in any medium, provided the original work is properly cited.

\begin{abstract}
This work describes a computational tool, based on an evolutionary algorithm, for the synthesis and optimization of submarine pipeline routes considering the incorporation of on-bottom stability criteria (OBS). This comprises a breakthrough in the traditional pipeline design methodology, where the definition of a route and the stability calculations had been performed independently: firstly, the route is defined according to geographical-topographical issues (including manual/visual inspection of seabed bathymetry and obstacles); afterwards, stability is verified, and mitigating procedures (such as ballast weight) are specified. This might require several design spirals until a final configuration is reached, or (most commonly) has led to excessive costs for the mitigation of instability problems. The optimization tool evaluates each candidate route by incorporating, as soft and hard constraints, several criteria usually considered in the manual design (pipeline length, bathymetry data, obstacles); also, with the incorporation of OBS criteria into the objective function, stability becomes an integral part of the optimization process, simultaneously handling minimization of length and cost of mitigating procedures. Case studies representative of actual applications are presented. The results show that OBS criteria significantly influences the best route, indicating that the tool can reduce the design time of a pipeline and minimize installation/operational costs.
\end{abstract}

\section{Introduction}

Submarine pipeline systems have been extensively used in the oil and gas industry, to transport the production between offshore platforms and/or to onshore processing facilities. Being one of the higher-cost items of the subsea layout, pipeline systems significantly affect the feasibility of an offshore project, thus demanding detailed studies to obtain efficient and low-cost designs, comprising an iterative and very complex process governed by several variables, following design recommendations addressed by codes such as DNVOS-F101 [1].

In this context, perhaps the most crucial step in the design of a submarine pipeline is the selection of its route. Traditionally, this task has been manually performed by experienced engineers, by inspecting the seabed bathymetry and available information regarding obstacles (including subsea equipment, flowlines, and other preexistent pipelines). Many environmental, commercial, regulatory, or even geopolitical issues may determine specific regions that should be avoided, 
for instance, corals, geotechnical hazards, or fields allotted to another oil company. There are many other variables that govern the selection of a route; thus the process has been treated almost as an "art," being highly dependent on the expertise of the engineer.

Previous works have already recognized [6-8] that the task of selecting a route with good performance and low cost could be automated by devising its formal description as a synthesis and optimization problem and building a computational tool based on evolutionary algorithms (EAs). Such algorithms have been successfully used in many complex engineering problems and have been shown to be useful for the optimization of offshore engineering problems [9-14].

In $[15,16]$ we have presented results of preliminary studies related to the development and implementation of a computational tool for the synthesis and optimization of submarine pipeline routes, based on Genetic Algorithms (GAs) $[17,18]$. The modeling of the optimization problem as presented in $[15,16]$ included only the basic geographicaltopographical issues associated with the route geometry and with the seabed bathymetry and obstacles. Those issues were considered for the representation of a candidate route in the context of the GA and for its evaluation in terms of criteria incorporated into the objective function and constraints. These criteria were defined considering only the main aspects already involved in the manual selection of a route, including basically the total pipeline length, interference with obstacles, minimum radius of curvature, and declivity. In [16] special focus was dedicated to the study and assessment of different constraint-handling techniques, including the $\varepsilon$-constrained method $[19,20]$. Therefore, the developments presented in $[15,16]$ could be seen as leading to a computational tool that, although innovative in itself, merely automated the process of selecting an optimal route, following the traditional design guidelines.

Here, the focus is on incorporating technical/engineering criteria into the route optimization tool, related to the structural behavior of the pipe under hydrostatic and environmental loadings: specifically, the implementation of on-bottom stability (OBS) criteria. The stability of a pipe is reflected by its ability to remain within its outline in the installed position (taking into account allowable tolerances) under environmental conditions of wave and current. The DNVRP-F109 code [21] presents criteria to check if a given pipe is stable or else to define minimal values of submerged weight to reach stability. This idea of incorporating OBS criteria into the route optimization tool comprises a breakthrough in the traditional pipeline design methodology and has originally been proposed in [22] where a preliminary implementation was sketched with the OBS criteria incorporated as constraints handled by the classic static penalty technique. This way, taking predefined values for the pipe submerged weight, the "optimal" route would seek areas and trajectories where the stability of the pipe is favored, according to the predominant direction of the environmental loadings.

However, that approach would possibly lead to longer routes and require a "trial-and-error" process, where the designer should perform successive runs of the optimization tool increasing the pipe weight in order to obtain smaller routes. Now, this work describes an improved approach to incorporate the OBS criteria into the optimization tool, where an additional term is introduced into the objective function, representing the ballast weight as an additional variable to be optimized. Thus, the weight required for stability at each pipe segment is directly provided as a result of an optimization run.

In the optimization procedure, candidate routes are represented by specific geometric parameterization and are evaluated in terms of several criteria incorporated in the objective function and in the constraint functions. These comprise the core of the route optimization model, which will be described in Section 2 that focuses on the route parameterization and encoding in the context of the GA; Section 3 presents the final form of the objective function and describes specifically the incorporation of the OBS criteria; Section 4 presents all remaining criteria that comprise the constraint functions (including their distinction between soft and hard constraints according to the consequences of their violation). Case studies are presented in Section 5 to illustrate the use of the optimization tool and assess the influence of the stability criteria on the definition of the optimal pipeline route. Lastly, final remarks and conclusions are presented in Section 6.

\section{Modeling and Solving the Route Optimization Problem}

2.1. Objective Function. A general constrained optimization problem is formally defined in an $n$-dimensional search space $S$ comprised by a vector of design variables $\mathbf{x}=$ $\left(x_{1}, x_{2}, x_{3}, \ldots, x_{n}\right)$. The following expression mathematically defines the problem:

$$
\begin{array}{cl}
\text { minimize } & f(\mathbf{x}) \\
\text { subject to } & g_{i}(\mathbf{x}) \leq 0, \quad i=1, \ldots, m \\
& h_{j}(\mathbf{x})=0, \quad j=1, \ldots, p \\
& l_{k} \leq x_{k} \leq u_{k}, \quad k=1, \ldots, q .
\end{array}
$$

The goal is to minimize an objective function $f(\mathbf{x})$, considering inequality and equality constraints (resp., $g_{j}(\mathbf{x}) \leq 0$ and $\left.h_{j}(\mathbf{x})=0\right)$ that define the feasible region. The components $x_{i}$ may have lower and upper bounds $\left[l_{k}, u_{k}\right]$. In engineering problems, the constraints are defined in terms of appropriate design criteria. As will be seen later, in the case of the pipeline route optimization problem these criteria may be expressed as inequality constraints $g_{j}(\mathbf{x})$ only, so equality constraints are not considered in this particular engineering application.

Here it is assumed that the outer diameter and wall thickness of the pipe segments have already been selected from previous design steps. Usually, the outer diameter is dictated by the amount of oil or gas to be transported, according to the yield of the well; and the wall thickness is dictated by strength constraints related to collapse under external hydrostatic pressure [23]. Thus, amongst the relevant 


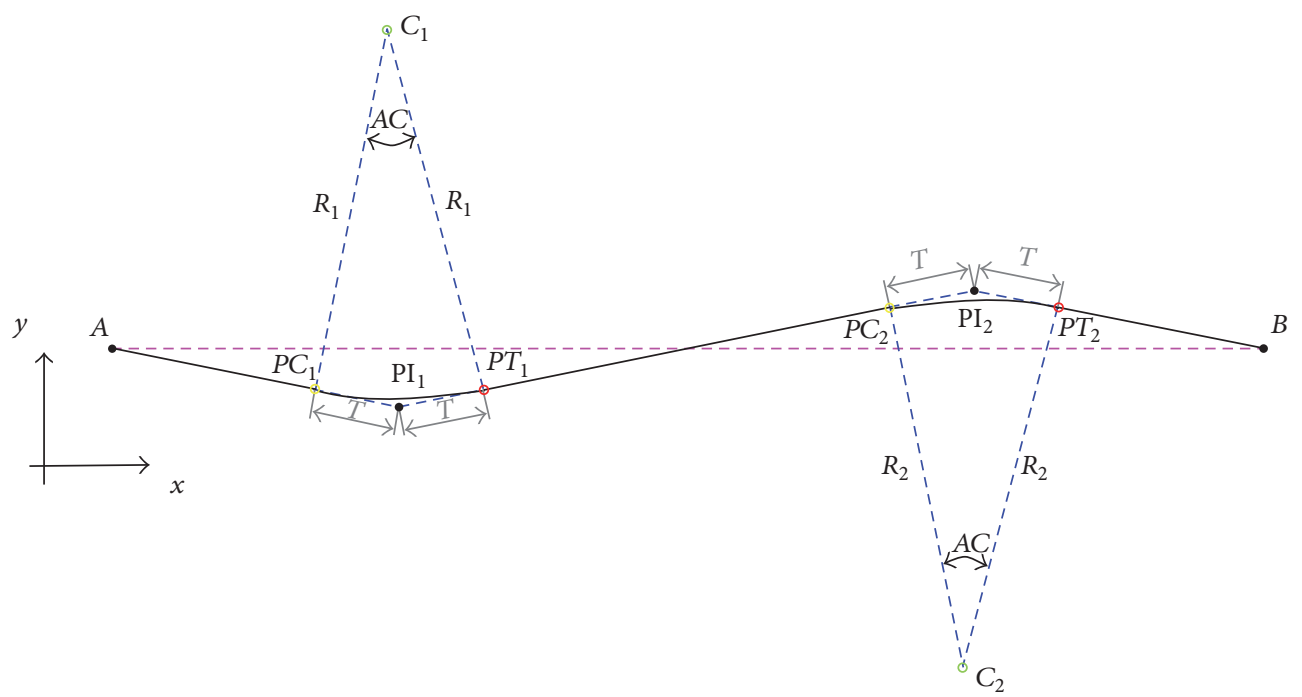

FIGURE 1: Planar representation of a route.

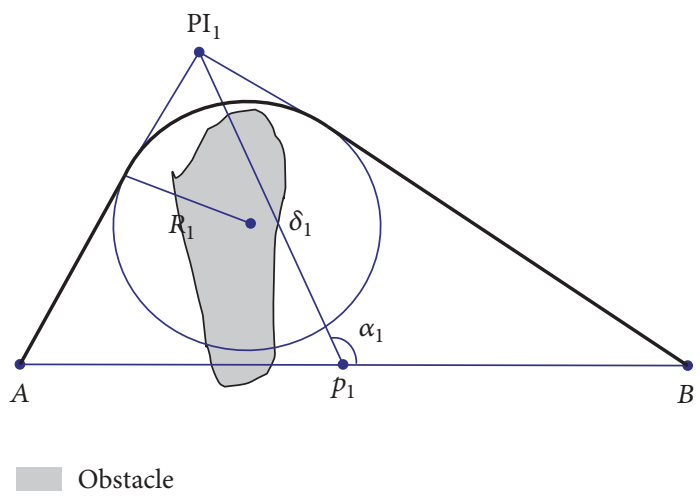

FIGURE 2: Primary parameters: curvature radius $\left(R_{i}\right)$; coordinates of PI $\left(\delta_{i}, \alpha_{i}\right)$.

variables that should be considered in the design of a pipeline route, the first factor that comes to mind is the total length, which should be minimized to reduce material and installation costs. Therefore, as presented in [16], the objective function $f$ could be defined simply as the ratio between the lengths of a given candidate route $\left(L_{\text {route }}\right)$ and of the straight line connecting endpoints $A$ and $B\left(L_{A B}\right)$ :

$$
f(\mathbf{x})=\frac{L_{\text {Route }}}{L_{A B}} .
$$

Considering the focus of the present paper which is to incorporate OBS criteria into the optimization procedure, later in Section 3 we will introduce an additional term into this objective function to take into account the weight of ballast that would be required to assure the stability of the pipeline. Now, before describing the other design criteria that comprise the constraints, in the remainder of this section we will describe the geometric representation of each candidate route in the context of the GA.
2.2. Geometric Parameterization of a Route. The modeling of the route optimization problem requires geometric parameterization to allow the representation of each individual candidate solution. The full formulation for this parametrization has already been detailed in [16]; here we will present only a brief overview. Figure 1 illustrates the planar representation of a route between endpoints $A$ and $B$, as a horizontal line defined in the $x y$-plane and comprised by a sequence of straight lines and curves; the curved segments are defined as circular arcs. Figure 2 illustrates the three primary parameters associated with each curve that, as demonstrated in [16], completely describe this representation:

(a) the curvature radius $\left(R_{i}\right)$ of each curve;

(b) the radial $(\delta)$ and angular $(\alpha)$ polar coordinates of Points of Intersection $\left(\mathrm{PI}_{i}\right)$-at which the prolongation of the straight lines (before and after each curve) intersects. These coordinates are relative to base points $\left(p_{i}\right)$ uniformly distributed along the straight line $A B$, as illustrated in Figure 2 showing the position of one $\mathrm{PI}_{i}$ associated with its corresponding base point $p_{i}$. 


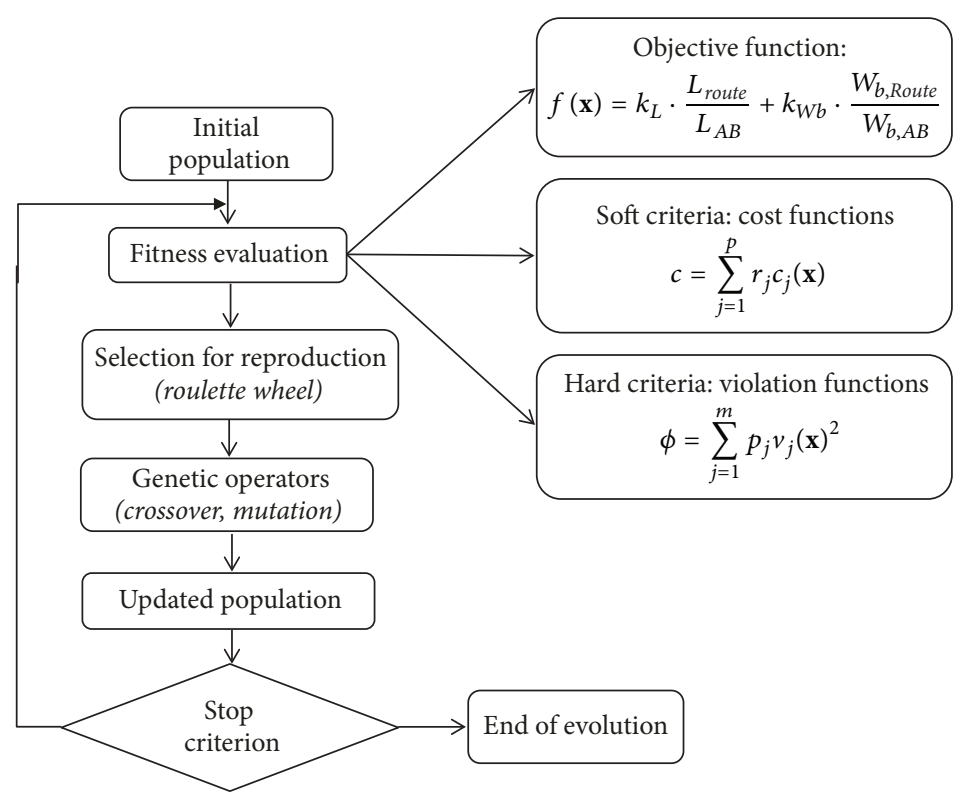

FIgURE 3: Schematic view of the main algorithm.

To obtain the complete three-dimensional representation of the route, in terms of the $(x, y, z)$ coordinates of a series of nodal points along each candidate route, the optimization tool incorporates facilities to import bathymetric data for a given subsea region. Such data are generated by specialized oceanographic vessels equipped with side-scan sonars and are usually available from design databases maintained by oil companies. They are provided as contour maps of isobathymetric curves, or isobaths. Having imported the isobaths, a standard gridding technique is then employed to generate a wireframe of quadrangular elements. Along the optimization process, the planar representation of each candidate route is divided into $N$ equal-length segments connecting $N+1$ nodal points. The $(x, y)$ coordinates are determined using the formulation mentioned above and described in detail in [16]. From these $(x, y)$ coordinates, the corresponding vertical $z$-coordinate is then obtained by interpolating on the quadrangular wireframe mesh.

2.3. Encoding the Routes into the GA. The pipeline route optimization tool described in this work employs canonical GA implementation. Each individual candidate route is encoded with real value representation in a chromosome with $n$ sets of genes. Each set is associated with a curve and comprises four genes. The last three genes correspond to the primary route parameters described above: radial coordinate $\delta_{i}$, angular coordinate $\alpha_{i}$, radius $R_{i}$; the first gene is an "activation key" $A_{i}$ that will be described shortly. The full codification of a chromosome can then be written as

$$
x^{r}=\left(A_{1} \delta_{1} \alpha_{1} R_{1} A_{2} \delta_{2} \alpha_{2} R_{2} \cdots A_{n} \delta_{n} \alpha_{n} R_{n}\right),
$$

where $A_{1} \delta_{1} \alpha_{1} R_{1}$ are the genes corresponding to the first curve and so on.

A population is represented by a set of $N$ individuals; in general, an initial population $P_{0}=\left\{x_{1}^{1}, x_{2}^{1}, \ldots, x_{N}^{1}\right\}$ is randomly created, where $x_{i}^{r}$ is the $i$ th individual in the $r$ th generation. Following Darwin's evolution theory, the fittest individuals have higher probability of surviving and reproducing, and their descendants keep the good genetic material in the species; thus, GAs involve mechanisms of natural selection, genetic recombination, and mutation. The "fitness" is provided by the evaluation of each individual via the objective and constraint functions. The individuals are selected for mating and reproduction by selection operators that generally follow probabilistic rules; here the fitnessproportional roulette wheel method is employed.

Mating is performed with crossover, combining genes from different parents to produce offspring and generate a new population. Here we consider single-point crossover, with the breakpoint on the parents' chromosomes randomly set according to a probability value equal to 0.6 (following usual guidelines). Thus the offspring inherit features from each of the parents and may be submitted to mutation, which confers innovative characteristics to the individual and provide a better exploration of the search space. Mutation alters each bit randomly with a relatively small probability; in the present implementation the mutation rate is set to 0.2 .

The implementation of the GA follows a generational approach, where the population is updated by replacing all parents by their offspring, which are made to compete with each other. Also, here we consider one-individual elitism; that is, the fittest individual from the previous population is directly injected into the new population. The process ends when a predefined stopping criterion is reached, and the individual with the best fitness is then defined as the solution of the optimization problem.

Figure 3 presents a schematic view of the basic steps of the GA, including the main expressions that will be employed 

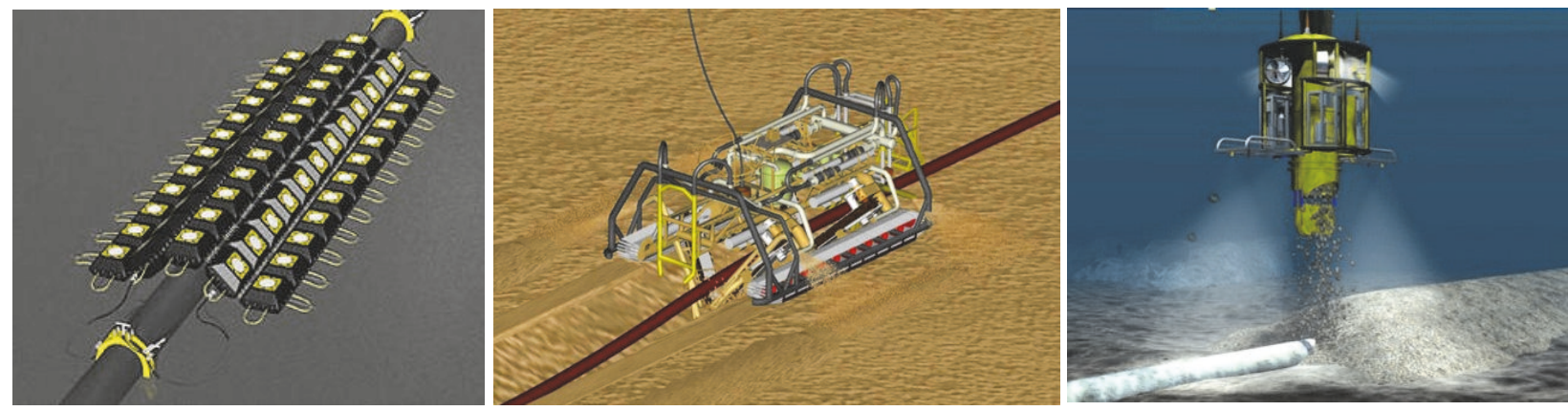

Figure 4: Procedures to stabilize a pipeline [2-4].

to evaluate each individual candidate route, that is, the objective, cost, and violation functions. Those expressions will be defined later in Sections 3.1 and 4.2.

\subsection{Selective Activation of Curves. The number of curves} needed to adequately represent a route may vary, depending on its length and the complexity of the scenario (in terms of seabed bathymetry and obstacles). Therefore, besides the three parameters for each curve described above (radius $R_{i}$; coordinates of PI: $\left.\delta_{i}, \alpha_{i}\right)$, the encoding of the route into the GA incorporates a fourth parameter: the "activation key" $A_{i}$. With this parameter, the number of curves actually employed to define each candidate route varies along the evolution process-from zero, corresponding to the trivial straight line $A B$, up to a user-defined maximum value $n_{\max }$. That is, the number of curves and their associated PI is also a variable of the optimization process.

During the generation of the first population (with individuals randomly created), a real value for the activation key $A_{i}$ corresponding to each curve in a route is randomly generated within the range $[0,1]$. This value indicates whether the corresponding curve is active or not, associated with a given user-defined activation threshold $A_{t}$ that defines the probability of activation of the curve as $\left(1.0-A_{t}\right) \times 100$. Then, these $A_{i}$ values are compared with the activation threshold $A_{t}$. If $A_{i}>A_{t}$, the corresponding curve is generated by the other three parameters: its radius and the polar coordinates of the PI relative to the closest base point. Otherwise, the curve is inactive and the corresponding section of the route is straight.

For instance, if the user feels that a given scenario is relatively simple, the user may define a higher value for the threshold parameter; for instance, $A_{t}=0.6$; this means that the probability of a given curve to be generated is only $40 \%$, and therefore the generated candidate routes will tend to have fewer curves. On the other hand, the user may force all $n_{\max }$ curves to be permanently active for all routes, simply by defining $A_{t}=0.0$. As the evolution of the GA proceeds, the selection/reproduction operators of the GA propagate the values of the activation keys $A_{i}$, favoring routes with either fewer or more curves depending on the complexity of the scenario; also, the mutation operator may introduce new random values for these genes.

\section{On-Bottom Stability Criteria}

3.1. Objective Function considering OBS. Now, to incorporate the OBS criteria into the optimization procedure, an additional term is introduced into the objective function of (2). The goal is to guide the optimization process towards an optimal solution that complies with the on-bottom stability with lower intervention costs. This term is defined as the weight of ballast required to stabilize a candidate route $W_{b \text {, Route }}$, normalized by the ballast $W_{b, A B}$ required to stabilize the straight route $L_{A B}$. With this additional term, the objective function now reads as follows:

$$
f(\mathbf{x})=k_{L} \cdot \frac{L_{\text {Route }}}{L_{A B}}+k_{W b} \cdot \frac{W_{b, \text { Route }}}{W_{b, A B}} .
$$

Usually those two objectives (minimizing length and ballast weight) may be conflicting: routes with shorter lengths may require more ballast weight, and vice versa. Thus, weighting factors $k_{L}$ and $k_{W b}$ are inserted so that the user can specify a relative measure of the importance of minimizing, respectively, length and ballast. As will be seen later in the results of the case studies, this feature presents a crucial role in the definition of an optimal route-recalling that there are different alternative procedures to provide ballast weight (including concrete mattress, trenching, burying, or rock dumping as illustrated in Figure 4); in a preliminary design stage (which is the focus of this optimization tool), actual data regarding the costs of those different procedures to provide ballast may not be easy to obtain. This way, the weighting factors allow the user to adjust the relative importance of each term of the objective function (length and ballast), favoring the minimization of either the pipeline length or the ballast weight, or even obtaining an intermediate solution. This allows the decision-maker to obtain different optimal routes that satisfy the conflicting objectives of the optimization. This feature will be illustrated later in the case studies.

The remainder of this section will begin by briefly summarizing, in Section 3.2, the main concepts behind the OBS criteria as defined in [21], focusing on the two design methods that will be incorporated into the optimization tool. It will be seen that such methods depend on several parameters related to not only the pipe segments themselves, but also the soil properties and environmental loads. All 
those parameters may vary along each candidate route and along the spatial domain it occupies on the seabed; thus, to consider these variations, each route is discretized into a given number of nodes (nNodes) and segments ( $n$ Segm). In Section 3.3, the OBS expressions of [21] will be rearranged to provide, for each segment, the ballast weight (per unit length) $w_{b \text {,pipe }}$ required to stabilize the pipe (either directly, or using iterative procedures to comply with the selected safety factors). The expressions will also be extended to consider slopes in irregular seabed. Then, the total ballast weight for the entire route $W_{b \text {, Route }}$ (to be incorporated into (4)) may be obtained by a summation for all pipe segments:

$$
W_{b, \text { Route }}=\sum_{i=1}^{n \text { Segm }} w_{b, \text { pipe }}(i) \cdot L_{\text {segm }}(i) \text {. }
$$

3.2. Approaches for the Analysis and Verification of OBS. The Recommended Practice DNV-RP-F109 [21] describes three different methodologies for analysis and verification of the lateral stability of a given pipeline configuration: (1) "absolute lateral static stability" with zero displacement, that is, ensuring that the hydrodynamic loads acting on the pipe are less than the soil resistance and that the vertical lift load is lower than the submerged weight; (2) "generalized lateral stability method," ensuring "no break-out" for a "virtually stable" pipe, allowing small displacements (less than about one-half diameter), taking advantage of the passive resistance of the soil and ensuring that the pipe does not move out of its cavity, with maximum displacements independent of time; and (3) "dynamic lateral stability analysis," allowing "accumulated displacements," with the pipe able to break out of (and return to) its cavity, and the soil resistance is dependent on the time-history of pipe displacements.

These approaches may be associated with analytical expressions, precalibrated curves, or dynamic FE analyses. In the case of the absolute lateral static stability method, static analytical expressions provide safety factors associated with the ratio between hydrodynamic loads and horizontal soil resistance; in the case of the generalized lateral stability method, precalibrated curves provide the minimum required weight for a given maximum allowable displacement.

Finally, the more complex dynamic lateral stability anal$y$ sis method is based on the generation of numerical models and the execution of dynamic analyses under environmental loadings of current and irregular wave, for each specific pipe configuration, taking into account the appropriate soil resistance forces. Differently from the previous methods, it does not directly provide specific design values (in terms of safety factors or required weight); rather, it provides results regarding the behavior of the pipeline, in terms of motions and stresses. These results should then be compared with the limit values of the respective design criteria, in order to obtain the corresponding safety factors.

In this work, we will study the implementation of the first two methodologies (absolute static stability and generalized stability) into the route optimization tool. The third approach is more adequate for more advanced stages of the design of the pipeline; its implementation would not be feasible in the context of the optimization tool, since it would require the dynamic analysis of each candidate route by a complete model in a finite element simulation program, demanding excessively high CPU costs.

3.2.1. Absolute Lateral Static Stability. According to [21], "absolute lateral static stability" is assured when both of the following conditions are met, respectively, for the lateral and vertical directions, considering a given safety factor $\gamma_{\mathrm{SC}}:$

$$
\begin{aligned}
\gamma_{\mathrm{SC}} \cdot \frac{F_{Y}^{*}+\mu \cdot F_{Z}^{*}}{\mu \cdot w_{s}+F_{R}} & \leq 1.0, \\
\gamma_{\mathrm{SC}} \cdot \frac{F_{Z}^{*}}{w_{s}} & \leq 1.0 .
\end{aligned}
$$

The first condition corresponds to the static lateral equilibrium, where the ratio between the horizontal components of the hydrodynamic loads and the soil resistance is checked against a safety factor $\gamma_{\mathrm{SC}}$. Hydrodynamic loads are incorporated in the horizontal force $F_{Y}^{*}$ as well as the vertical lift force $F_{Z}^{*}$, all calculated by the Morison formula taking the current velocity at the level of the pipe and the water velocity obtained by a linear wave theory considering a single regular wave component. In [21] specific considerations are presented for the use of the Morison formula, in terms of current velocity reduction factors to take into account the soil rugosity $z_{0}$ and load reduction factors to take into account the pipe-soil interaction (in terms of the soil permeability, pipe penetration, and trenching). The soil resistance is calculated in two parts: one proportional to the normal force acting on the soil, being determined by a friction coefficient $\mu$ affecting both the lift force $F_{Z}^{*}$ and the pipe submerged weight $w_{s}$, and another that corresponds to the passive resistance $F_{R}$ due to an initial penetration of the pipe.

The second condition, which corresponds to the static vertical equilibrium, simply checks the ratio between the lift force $F_{Z}^{*}$ and the pipe weight $w_{s}$ against the same safety factor $\gamma_{\mathrm{SC}}$.

3.2.2. Generalized Lateral Stability. The generalized lateral stability method described in Section 3.5 of [21] is a relatively more complex and less conservative method. Differently from the absolute lateral static stability method, it now allows some pipe displacement, under the action of a design spectrum of oscillatory wave-induced velocities $U_{s}$ at the pipeline level.

This method depends on the level of allowable displacement, specified as a value that does not result in excessive pipe deformations or stresses. Two levels of allowable displacements are suggested: (a) up to one-half pipe diameter (corresponding to the "virtually stable pipe") and (b) up to ten diameters. According to the desired level, the method provides values for the weight required for global stability of the pipeline, in terms of a significant weight parameter L: that is, $L_{\text {stable }}$ and $L_{10}$, respectively. Intermediate displacement criteria can be established by defining the required weight $L_{Y}$ 


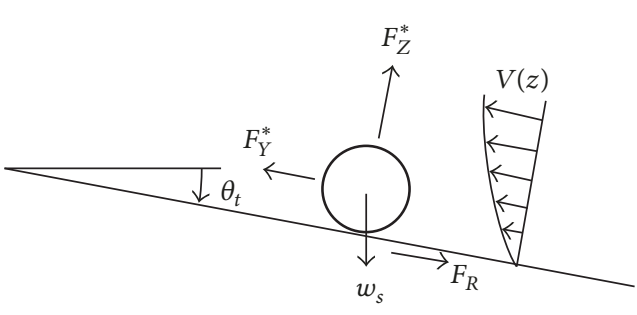

(a) Up slope velocity

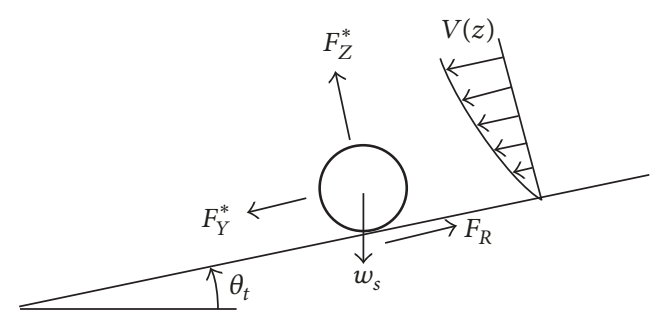

(b) Down slope velocity

FIgURE 5: Hydrodynamic Forces considering slopes (adapted from [5]).

for an allowable displacement $Y$ according to the following formula:

$$
\begin{aligned}
\log \left(L_{Y}\right)= & \log \left(L_{\text {stable }}\right)+\frac{\log \left(L_{\text {stable }} / L_{10}\right)}{\log (0.5 /(0.01 \cdot \tau))} \\
& \cdot \log \left(\frac{Y}{0.5}\right),
\end{aligned}
$$

where $\tau$ is the number of oscillations in the design bottom velocity $=T / T_{u}$ (with $T$ being the wave period and $T_{u}$ the spectrally derived mean zero up-crossing period).

Values for the parameters $L_{\text {stable }}, L_{10}$ are obtained through empirical expressions and design curves, calibrated from a database of results for a large number of dynamic analyses. A detailed description can be found in [21]; in summary, they depend on a set of dimensionless parameters, including parameters related to the type of soil (clay or sand) and to the wave and current loadings (spectrally derived values of the oscillatory fluid flow due to waves and steady current velocity). To check if a pipe is stable, the required value for the significant weight parameter $\left(L_{\text {stable }}, L_{10}\right.$, or $\left.L_{Y}\right)$ given by the design curves of [21] may be compared with the $L$ parameter calculated for the pipe by the following expression [21]:

$$
L=\frac{w_{s}}{0.5 \cdot \rho_{w} \cdot D \cdot U_{s}^{2}},
$$

where $\rho_{w}$ is the mass density of water, $D$ is the external pipe diameter, and $U_{s}$ is the spectrally derived oscillatory velocity (significant amplitude) for design spectrum, perpendicular to the pipeline. If the value calculated by expression (8) is higher than the required value $\left(L_{\text {stable }}, L_{10}\right.$, or $\left.L_{Y}\right)$, then the pipe is stable.

3.3. Calculating the Ballast Terms of the Objective Function. The expressions presented in Sections 3.2.1 and 3.2.2 allow the verification of the OBS for a given pipeline configuration; in the context of a conventional design procedure, the engineer should then select a set of values for the pipe design parameters in order to obtain a minimum submerged weight that complies with those expressions. On the other hand, in the context of the optimization procedure, these expressions may be rearranged to provide safety factor values for each pipe segment into which a candidate pipeline route has been discretized; in some cases, they may provide directly the ballast weight required to stabilize the pipe.

The "absolute lateral static stability" expressions (6) may be rearranged to provide values for the lateral and vertical safety factors $\gamma_{\mathrm{SC}_{y}}$ and $\gamma_{\mathrm{SC}_{z}}$, respectively, as follows:

$$
\begin{aligned}
& \gamma_{\mathrm{SC}_{y}}=\frac{\mu \cdot w_{s}+F_{R}}{\left|F_{Y}^{*}+\mu \cdot F_{Z}^{*}\right|}, \\
& \gamma_{\mathrm{SC}_{z}}=\frac{w_{s}}{F_{Z}^{*}} .
\end{aligned}
$$

Similarly, the "generalized lateral stability" expressions that define the different "significant weight parameters" $L_{\text {stable }}, L_{10}$, or $L_{Y}$ may be combined to provide a safety factor $\gamma_{\mathrm{SC}_{y}}$ as the ratio between the significant weight parameter $L$ of the pipeline and the significant weight parameter $L_{\text {stable }}, L_{10}$, or $L_{Y}$ required to obtain the stability; in the case of $L_{10}$, for instance, we have

$$
\gamma_{\mathrm{SC}_{y}}=\frac{L}{L_{10}} .
$$

The OBS expressions described above were originally derived in DNV-RP-F109 [21] considering only horizontal seabed. However, since the optimization tool considers irregular seabed defined by actual bathymetric data, it is desirable to employ expressions that take into account the declivity of the bathymetric floor transversal to the pipe axis (Figure 5). This allows the optimization process to favor relatively leveled trajectories (perpendicular to the pipe), to avoid the sliding of the pipe on regions with high transversal slopes.

To reach this goal, (9) that provides the lateral safety factor $\gamma_{\mathrm{SC}_{y}}$ is altered to take into account the transversal declivity angle $\theta_{t}$ as follows:

$$
\gamma_{\mathrm{SC}_{y}}=\frac{\mu \cdot w_{s} \cdot \cos \left(\theta_{t}\right)+F_{R}}{\left|F_{Y}^{*}+w_{s} \cdot \sin \left(\theta_{t}\right)+\mu \cdot F_{Z}^{*}\right|} .
$$

A similar expression for the vertical safety factor $\gamma_{\mathrm{SC}_{z}}$ may be derived from (10):

$$
\gamma_{\mathrm{SC}_{z}}=\frac{w_{s} \cdot \cos \left(\theta_{t}\right)}{F_{Z}^{*}} .
$$


Regarding the generalized stability method, the following expression can be defined to provide a safety factor considering slopes:

$$
\gamma_{\mathrm{SC}_{y}}=\frac{L \cdot \cos \left(\theta_{t}\right)}{L_{10}} .
$$

Taking these expressions for the safety factors, a simple iterative procedure has been devised to provide the ballast weight per unit length $w_{b \text {,pipe }}$ required to stabilize each pipe segment. Basically, this procedure gradually increases an initial estimate for $w_{b \text {,pipe }}$ until it provides a safety factor that meets a given minimum safety factor $\gamma_{\mathrm{SC}}$, such as those listed in [21]. Then, the corresponding values for all $n$ Segm pipe segments are summed to obtain the total ballast weight for the entire route $W_{b \text {, Route }}$, as indicated in (5). The ballast $W_{b, A B}$ required to stabilize the straight route $L_{A B}$ (that nondimensionalizes the OBS term of (4)) is also calculated following a similar procedure.

Considering particularly the "generalized lateral stability" method, the expressions that define the different "significant weight parameters" ( $L_{\text {stable }}, L_{10}$, or $\left.L_{Y}\right)$ might provide directly the submerged weight of the pipe (per unit length) required for stability-simply by equating (for instance) $L_{10}=L$ and then rearranging (8) to obtain the total required submerged weight, from which the desired ballast weight per unit length may be obtained by subtracting the original pipe weight. However, strictly speaking this would apply only for procedures that are not influenced by the calculation of hydrodynamic loads, which depend on the outer diameter $D$ of the pipe; that is, concrete mattress, trenching, burying, or rock dumping as illustrated in Figure 4. For the most usual procedures to apply ballast weight to a pipe, which are based on installing an additional layer of concrete around the pipe itself, two terms of (8) would be simultaneously affected ( $D$ and $w_{s}$ ); to solve this issue and provide the desired value for $w_{b \text {,pipe }}$, an iterative procedure similar to that described above is also applied.

\section{Other Design Criteria: Constraints}

4.1. Design Criteria. Besides the total length and the OBS criteria that have been incorporated into the objective function as described above, the route optimization tool incorporates several other design criteria related to geographical/topographical aspects associated with the seabed bathymetry (such as slopes and interference with obstacles). These criteria and their respective violation functions had already been described in detail in [16]; in the remainder of this section we will present only a brief summary.

4.1.1. Interference with Obstacles. Besides automatically importing isobathymetric curves from design databases to generate the three-dimensional representation of the route (as described in Section 2.2), the tool also incorporates facilities to selectively or fully import data describing the positioning and specification of subsea obstacles that should be avoided. Such data is gathered by specialized vessels equipped with a ROV (remotely operated vehicle), including also geophysical and geotechnical data obtained from the bathymetry and sonography, with information about facies and geotechnical hazards that identify critical areas such as geological faults, rocky outcrops, natural reefs.

Thus, different types of obstacles may be considered: subsea equipment, wellheads, flowlines, other preexistent pipelines, regions with corals or geohazards that should be avoided. To each one the user may associate a level of severity, according to the consequences of the possible interference: Level 0 , tolerated, with low severity and easily mitigated; Level 1, conditionally allowed, with moderate severity introducing relatively higher mitigation costs; Level 2, not allowed, with severity being high; Level 3, not allowed, with severity being critical. During the optimization process the number intersections for each severity level is counted and stored in variables nInterS and nInterH (according to the classification of the interference as soft and hard, as will be seen later); interference is identified by verifying the intersections between the segments or volumes that define the obstacles, against nodal points and segments along each candidate route.

4.1.2. Self-Crossing. The geometric representation described in Section 2.2 might eventually lead the optimization process to generate routes in which the pipeline passes over itself. To identify such configurations with self-crossings or loops, the optimization tool incorporates an algorithm that spans all nodes and segments of the route and counts the number of self-crossings ( $n$ SelfCross).

4.1.3. Minimum Length of Straight Sections. Pipeline launching operations require straight sections between two consecutive curves, to allow proper space for the maneuvering of the launching ship. Straight sections are already incorporated in the geometric parameterization described before, but if a given minimum length $L_{\text {min }}$ is not respected, the operation may be infeasible. To identify such situations, the length $L_{i}$ of all straight sections (nStraight, including the first and last at the beginning/end of the route) is checked against this limit $L_{\text {min }}$.

4.1.4. Minimum Radius of Curvature. During the pipeline launching operation, the top connection device at the launching ship applies a given tension to the pipe. After the completion of the operation and the accommodation of the pipeline on the seabed, the pipe segments still maintain a certain level of residual tension. It is the balance between this residual tension and the lateral friction forces from the soil that maintains the curved sections of the pipeline over the predefined route. On curves with smaller radius the friction forces may not be sufficient, and the pipe can slide sideways. Therefore, to assure the feasibility of a given route, the curved sections should present radius of curvature larger than a given value $R_{\min }$, function of the pipe-soil friction coefficient $\mu$, pipe weight $w_{s}$, and residual pipe tension $T_{\text {residual }}$ at its equilibrium configuration after installation. 
To identify such situations, the optimization tool spans each node of the route along the curved sections, calculates the required limit $R_{\min }$, and checks this limit against the radius of the curve $R_{i}$. Being a function of the soil and pipe parameters that are also variable, $R_{\min }$ may vary from curve to curve, or even along each curve.

4.1.5. Declivity. The slope of the regions where the pipeline is set also comprises a design criterion for the definition of the route. Values for the longitudinal declivity $\theta_{L}^{i}$ along the nodes of a candidate route ( $n$ Nodes) can be calculated taking the $z$ coordinate of two consecutive nodes, which in turn are determined from the grid of quadrangular elements interpolated from the isobathymetric curves. To implement this criterion, the optimization tool incorporates an algorithm that check those declivities $\theta_{L}^{i}$ against a user-defined limit $\theta_{\text {LLim }}$.

4.1.6. Attractors. During the design of a pipeline route, it may be desirable to keep the route near some regions of interest, referred here as "attractors," for instance, a manifold connecting other pipelines or flowlines, or even production platforms, allowing the pipeline to collect the production of adjacent oil fields. To identify such configurations, the tool incorporates an algorithm that, for a total number of $n A t t r$ attractors, calculates the distance between the route segments closest to a given attractor $\left(\operatorname{minDist}{ }^{i}\right)$ and checks if this value is smaller than a user-defined limit expressed as the radius of the "attractor circle" $R_{\text {Attr }}{ }^{i}$.

4.2. Soft and Hard Criteria: Handling of Constraints. All the aforementioned design criteria should be incorporated as constraints into the optimization process. Since GAs and other evolutionary algorithms were originally designed to deal with unconstrained search spaces, specific constrainthandling techniques are required to guide the evolutionary search process to feasible regions.

Following the approach presented in [16], according to the consequences of their violation the criteria described above are classified as soft and hard, which will be handled separately using different techniques. The violation of the socalled soft criteria would not indicate that the corresponding solution is infeasible, since mitigation procedures could be adopted. This is the case, for instance, of interference with Level 0 and Level 1 obstacles, such as preexistent pipelines; in this case there are specific installation procedures that allow the new pipe to pass safely over the existing one. Solutions that violate these soft criteria would still be feasible, although requiring additional costs; therefore they do not formally characterize constraints in the search space.

On the other hand, violation of hard criteria cannot be mitigated and should be avoided. Their violation will mark the solution as infeasible, and therefore they should be formally considered as constraints, to be treated by a particular constraint-handling technique. Amongst the criteria listed in Section 4.1, the ones that are classified as hard are interference with Level 2 and Level 3 obstacles (such as subsea equipment or mooring lines), self-crossing, minimum length of straight sections, minimum radius of curvature.
4.2.1. Soft Criteria. Besides interference with Level 0 and Level 1 obstacles, the longitudinal declivity and attractors may be considered as soft criteria; in the former case, mitigation procedures can be adopted for route segments with declivity exceeding the specified limit. Attractors can also be considered a soft criterion if one assumes that routes passing far from such regions would not be infeasible but would require higher costs.

The soft criteria are handled by adding, to the objective function of (4), dimensionless cost functions $c_{j}(\mathbf{x})$ associated with the violation of the $j$ th soft criterion, weighted by positive constant values $r_{j}$ that provide a relative measure of the importance of the criterion. This is equivalent to the classic static penalty technique and leads to the following expanded expression for the objective function $f$ :

$$
\begin{aligned}
f(\mathbf{x})= & k_{L} \cdot \frac{L_{\text {route }}}{L_{A B}}+k_{W b} \cdot \frac{W_{b, \text { Route }}}{W_{b, A B}} \\
& +\left.\sum_{j=1}^{p} r_{j} c_{j}(\mathbf{x})\right|_{\text {SoftCriteria }} .
\end{aligned}
$$

This objective function corresponds to a minimization problem, where the goal is to reduce the value of $L_{\text {route }}$, the weight of ballast $W_{b, \text { Route }}$, and the cost terms related to the soft criteria. For the ideal route one would trivially have $L_{\text {route }}=$ $L_{A B}, W_{b, \text { Route }}=0$, and $f=1$. Solutions that violate one of the soft criteria are not considered as infeasible; adding the cost terms only represents a handicap, to favor the other possible solutions that do not violate them. Mathematical expressions for the cost functions $c_{j}(\mathbf{x})$ associated with those soft criteria will be provided shortly in Section 4.2.3.

4.2.2. Hard Criteria: The $\varepsilon$-Constrained Method. The hard criteria that formally characterize constraints are handled by the $\varepsilon$-constrained method $[19,20]$. In this technique, the individuals are ranked based on a lexicographic order of sets of values $(f, \phi)$ assigned to each individual, where $\phi$ is a function that indicates the level of constraint violation and $v_{j}(\mathbf{x})$ are the violation functions associated with the jth hard criterion, weighted by positive constant values $p_{j}$ that provide a relative measure of the importance of each criterion:

$$
\phi=\sum_{j=1}^{m} p_{j} v_{j}(\mathbf{x})^{2},
$$

$v_{j}(\mathbf{x})>0$ if constraint is violated, $v_{j}(\mathbf{x})=0$ otherwise.

Considering two individuals 1 and 2, their ranking is performed as follows:

$$
\begin{aligned}
\left(f_{1}, \phi_{1}\right) & <\varepsilon\left(f_{2}, \phi_{2}\right) \\
& \mathbb{1} \\
f_{1}<f_{2}, & \text { if } \phi_{1}, \phi_{2} \leq \varepsilon, \\
f_{1}<f_{2}, & \text { if } \phi_{1}=\phi_{2}, \\
\phi_{1}<\phi_{2}, & \text { otherwise. }
\end{aligned}
$$


TABLE 1: Soft criteria and cost functions.

\begin{tabular}{|c|c|}
\hline Soft criteria & Cost function \\
\hline $\begin{array}{l}\text { Interference with Level } 0 \text { and Level } 1 \text { obstacles } \\
\text { Severity: weight factor assigned by the specialist to each level }\end{array}$ & $c_{\text {obst }}=\sum_{i=1}^{\text {nInterS }}$ Severity $_{i}$ \\
\hline $\begin{array}{l}\text { Longitudinal declivity } \\
\text { Activated for the nodes of the route with declivity } \theta_{L}^{i} \\
\text { greater than the limit } \theta_{L_{-} \text {Lim }}\end{array}$ & $c_{\text {decliv }}=\frac{\sum_{i=1}^{n \text { Nodes }} \theta_{L}^{i}-\theta_{L L i m}}{n \text { Nodes }}$ \\
\hline $\begin{array}{l}\text { Attractor } \\
\text { Encourage routes near regions of interest; activated whenever route does not pass } \\
\text { into the "attractor circle." } \\
w_{i}: \text { weight factor indicating relative importance of each attractor }\end{array}$ & $c_{\text {attr }}=\sum_{i=1}^{n A t t r} w_{i} \frac{\operatorname{minDist}^{i}}{L_{A B}}$ \\
\hline
\end{tabular}

TABLE 2: Hard criteria and violation functions.

\begin{tabular}{|c|c|}
\hline Hard criteria & Violation function \\
\hline $\begin{array}{l}\text { Interference with Level } 2 \text { and Level } 3 \text { obstacles } \\
\text { Severity: weight factor assigned to each level }\end{array}$ & $v_{\text {obst }}=\sum_{i=1}^{\text {nInterH }}$ Severity $_{i}$ \\
\hline Self-Crossing & $v_{\text {SelfCross }}=n$ SelfCross \\
\hline $\begin{array}{l}\text { Minimum length of straight sections } \\
\text { Activated whenever one or more straight sections have length } L_{i} \text { lower than } L_{\min }\end{array}$ & $v_{L \min S t r}=\sum_{i=1}^{n S t r a i g h t} \frac{L_{\min }-L_{i}}{L_{\min }}$ \\
\hline $\begin{array}{l}\text { Minimum radius of curvature } \\
\text { Activated whenever a curve has radius lower than } R_{\min }\end{array}$ & $\begin{array}{c}v_{R \min }=\sum_{i=1}^{n \text { Nodes }} \frac{\left(R_{\min }-R_{i}\right)}{R_{\min }} \\
R_{\min }=\frac{T_{\text {residual }}}{\mu * w_{s}}\end{array}$ \\
\hline
\end{tabular}

In this expression $\varepsilon$ is a specified value that represents tolerance related to the sum of constraint violation. The first option means that if both solutions in the pairwise comparison are feasible or slightly infeasible, as determined by the $\varepsilon$ value, they are compared using their objective function values $f$. The second and third options refer to infeasible solutions: the second option means that two infeasible solutions presenting the same sum of constraint violation $\phi$ will also be compared using their objective function values; otherwise, in the third option they will be compared based on their sum of constraint violation.

The objective function $f$ is given by $f(\mathbf{x})$ of (15) that incorporates the cost functions for the soft criteria. Also, assuming that the feasibility of a solution is more important than the minimization of its objective function, we take $\varepsilon=0$; in this case, the lexicographical ordering considers that the minimization of the sum of constraint violation $\phi$ precedes the minimization of the objective function $f$. Feasible solutions are ranked based on their unconstrained objective function values $f$; infeasible solutions are compared based on their sum of constraint violation $\phi$. If one of the solutions is infeasible $(\phi>0)$ and the other is feasible $(\phi=0)$, the latter will be selected.

4.2.3. Summary of the Soft and Hard Criteria. Tables 1 and 2 summarize, respectively, the soft and hard criteria along with their associated cost and violation functions $\left(c_{j}(\mathbf{x}), v_{j}(\mathbf{x})\right)$. Although the expressions for these functions may seem simple, their evaluation is not trivial since it involves calculations performed for possibly hundreds or thousands of points along each candidate route, using the three-dimensional representation that combines the planar formulation with the vertical $z$-coordinates determined by interpolating the seafloor bathymetric data as described in Section 2.2. Later in the presentation of the case studies, the values assigned for each parameter of these functions will be shown.

\section{Case Studies}

5.1. Description of the Scenarios. To assess the influence of the stability criteria on the definition of the optimal pipeline route, two different scenarios are considered. The first, illustrated in Figure 6, corresponds to a relatively simpler subsea layout where a pipeline is designed to connect two neighboring platforms, while the second (Figure 7) is associated with a complex layout, including more obstacles and also attractors. The straight line $A B$ connecting the points of the route to be optimized is depicted in pink. Levels $0,1,2$, and 3 obstacles are indicated, respectively, in green, yellow, red, and black.

For scenario 1 (Figure 6) only Level 2 obstacles are defined, the mooring lines of the floating platforms, indicated in red. For scenario 2 (Figure 7) the mooring lines are treated as Level 4 obstacles, indicated in black; Levels 0 and 1 obstacles are also included (preexistent pipelines and flowlines); and another Level 4 obstacle is added: the black polygon representing an area allotted to another oil company. 


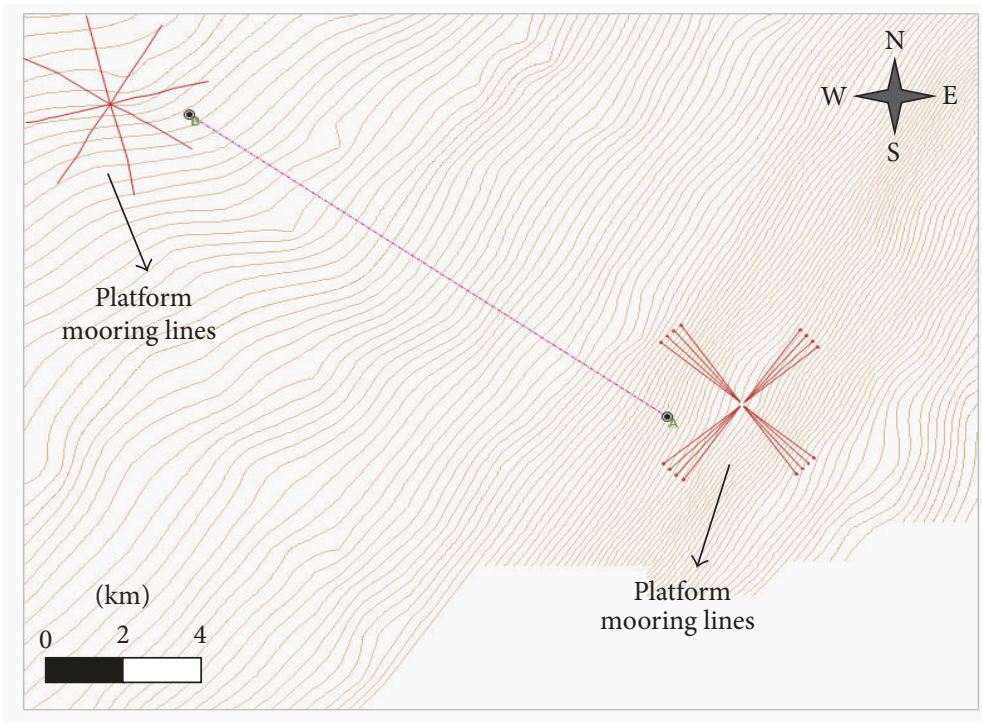

FIGURE 6: Scenario 1: simple layout.

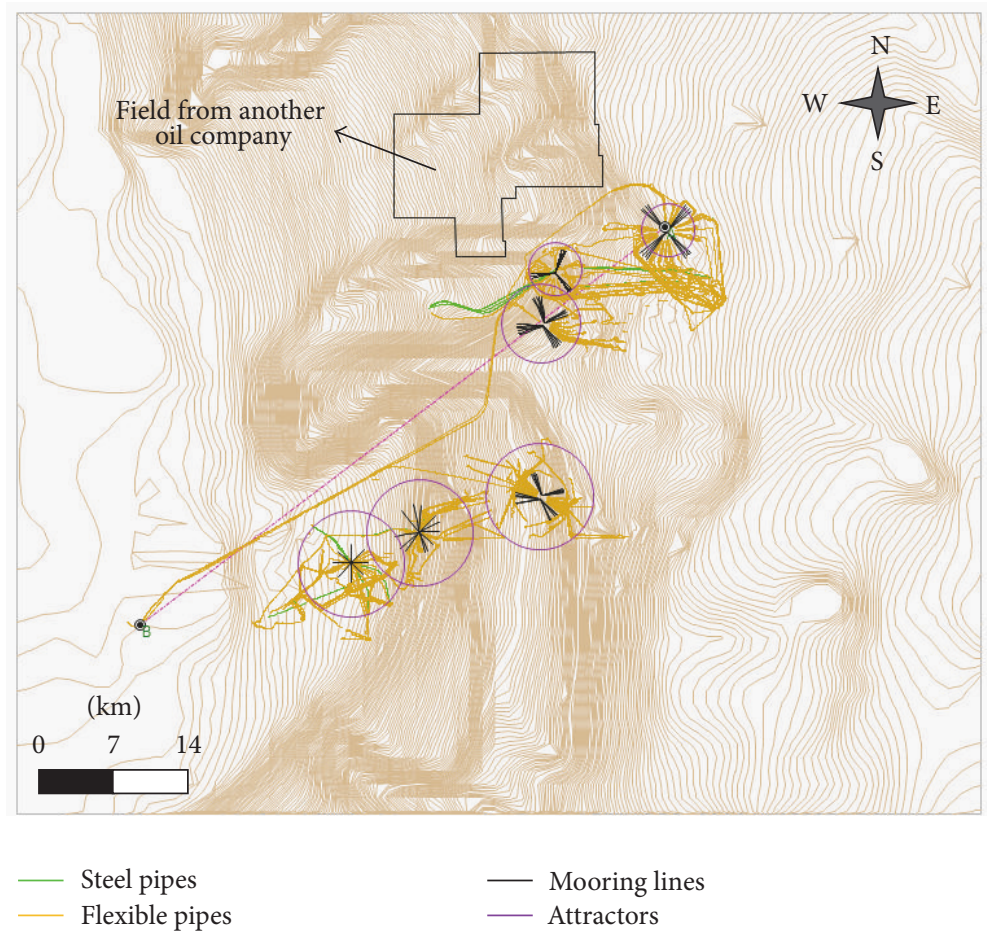

FIGURE 7: Scenario 2: complex layout with obstacles and attractors.

The "attractor" regions close to other platforms are indicated as purple circles.

Table 3 presents the main geometric parameters defining the endpoints of the routes for these scenarios, while Table 4 describes the properties of the pipeline for each scenario. Table 5 presents the values for the soil parameters required to evaluate the on-bottom stability criteria and also the minimum radius of curvature (see (26)). One can observe that scenario 2 comprises two different soil types, defined according to the water depth. The pipeline properties also vary with the water depth.

5.2. Objective Function and Constraints. Table 6 summarizes the main parameters of the optimization algorithm employed for each scenario described above: those that define the search space of the problem (the maximum number of curves $n_{\max }$ and the threshold of the activating factor $A_{t}$ as described in Section 2.4), along with the number of individuals in each 
TABLE 3: Main geometric parameters.

\begin{tabular}{lccc}
\hline Scenario & $\begin{array}{c}L_{A B} \\
(\mathrm{~km})\end{array}$ & $\begin{array}{c}\text { Depth of endpoint } B \\
(\mathrm{~m})\end{array}$ & $\begin{array}{c}\text { Depth of endpoint } A \\
(\mathrm{~m})\end{array}$ \\
\hline 1 & 12.359 & 14 & 529 \\
2 & 49.393 & 96 & 1782 \\
\hline
\end{tabular}

TABLE 4: Pipeline properties.

\begin{tabular}{|c|c|c|c|c|}
\hline Parameter & & Scenario 1 & \multicolumn{2}{|c|}{ Scenario 2} \\
\hline Outside diameter (OD) & & $323.85 \mathrm{~mm} / 12 \frac{3}{4}$ in & \multicolumn{2}{|c|}{$457 \mathrm{~mm} / 18$ in } \\
\hline \multirow{6}{*}{ Wall thickness $t_{s}$} & & \multirow{6}{*}{$19.05 \mathrm{~mm} / \frac{3}{4}$ in } & Depth (m) & Thickness \\
\hline & & & $0-300$ & $17.5 \mathrm{~mm} / \frac{2}{3}$ in \\
\hline & & & $300-800$ & $19.1 \mathrm{~mm} / \frac{3}{4}$ in \\
\hline & & & $800-1190$ & $22.2 \mathrm{~mm} / \frac{7}{8}$ in \\
\hline & & & $1190-1535$ & $25.4 \mathrm{~mm} / 1 \mathrm{in}$ \\
\hline & & & $1535-1800$ & $28.6 \mathrm{~mm} / 1 \frac{1}{8}$ in \\
\hline \multicolumn{3}{|l|}{ Steel specific weight $\rho_{s}$} & $77000 \mathrm{~N} / \mathrm{m}^{3}$ & \\
\hline \multirow{5}{*}{ Corrosion coating } & \multirow{3}{*}{ Thickness $\left(t_{p}\right)$} & \multirow{3}{*}{$76 \mathrm{~mm} / 3$ in } & Thickness & Spec. weight $\left(\mathrm{N} / \mathrm{m}^{3}\right)$ \\
\hline & & & $0.15 \mathrm{~mm} / 0.006 \mathrm{in}$ & 14126.4 \\
\hline & & & $0.20 \mathrm{~mm} / 0.008 \mathrm{in}$ & 8829 \\
\hline & \multirow[t]{2}{*}{ Spec. weight $\left(\rho_{p}\right)$} & \multirow[t]{2}{*}{$8826 \mathrm{~N} / \mathrm{m}^{3}$} & $3.65 \mathrm{~mm} / \frac{1}{7}$ in & 8829 \\
\hline & & & $48 \mathrm{~mm} / 1 \frac{8}{9}$ in & 8829 \\
\hline
\end{tabular}

TABLE 5: Soil properties.

\begin{tabular}{lccc}
\hline Parameter & Scenario 1 & Scenario 2 & 0-300 m \\
\hline Type & Sand & Sand & Clay \\
Pipe-soil friction coefficient $(\mu)$ & 0.7 & 0.6 & 0.2 \\
Dry unit soil weight $\left(\gamma_{s}\right)$ & - & - & $1800 \mathrm{~m}$ \\
Submerged unit soil weight $\left(\gamma_{s}^{\prime}\right)$ & $13500 \mathrm{~N} / \mathrm{m}^{3}$ & $13500 \mathrm{~N} / \mathrm{m}^{3}$ & - \\
Undrained clay shear strength $\left(s_{u}\right)$ & - & - & $10000 \mathrm{~N} / \mathrm{m}^{3}$ \\
\hline
\end{tabular}

TABLE 6: Parameters of the optimization algorithm.

\begin{tabular}{lcccc}
\hline & $N_{\text {pop }}$ & MaxGen & $n_{\max }$ & \\
\hline Scenario 1 & 60 & 200 & 4 & 0.15 \\
Scenario 2 & 100 & 300 & 6 & 0.15 \\
\hline
\end{tabular}

population $N_{p o p}$ and the maximum number of generations MaxGen comprising the termination criterion.

To provide a relative measure of the importance of minimizing, respectively, total length and ballast weight, different sets of optimization runs are performed varying the weighting factors $k_{L}$ and $k_{W b}$ employed in the objective function ((4) and (15)). Table 7 presents three sets of values: the first and third favors, respectively, the minimization of the pipeline length (possibly requiring more ballast weight) and vice versa, and the second corresponds to an intermediate value for the relative pipeline length weighting factor $\left(k_{L}\right)$.

The general form of the objective function is represented by (15), recalling that this expression incorporates the cost functions $c_{j}(\mathbf{x})$ for the soft criteria whose violation can be mitigated. The cost functions themselves were already presented in Table 1; now, Table 8 presents the cost factors $r_{j}$ and the other user-assigned values for the parameters associated with these soft criteria that will be employed in the 
TABLE 7: Weighting factors associated with the objective function.

\begin{tabular}{lccc}
\hline & Weighting factors Eq. (15) & \multicolumn{1}{c}{ Scenario } & 1 \\
\hline \multirow{2}{*}{ Favoring length } & $k_{L}$ & 50 & 10 \\
& $k_{W b}$ & 0.01 & 0.01 \\
Intermediate & $k_{L}$ & - & 5 \\
Favoring ballast & $k_{W b}$ & - & 1 \\
& $k_{L}$ & 0.01 & 1 \\
\hline
\end{tabular}

TABLE 8: Parameters of the cost functions associated with the soft criteria.

\begin{tabular}{|c|c|c|c|c|c|}
\hline \multirow{2}{*}{ Criterion } & \multicolumn{2}{|c|}{ Cost factors $r_{j},(15)$} & \multirow{2}{*}{ Parameter } & \multicolumn{2}{|r|}{ Values } \\
\hline & Scenario 1 & Scenario 2 & & Scenario 1 & Scenario 2 \\
\hline \multirow{3}{*}{ Interference with obstacles } & & & Severity values, (19) & & \\
\hline & 1 & 1 & Level 0 (green) & \multicolumn{2}{|r|}{0.2} \\
\hline & & & Level 1 (yellow) & \multicolumn{2}{|r|}{0.5} \\
\hline Declivity & 5 & 1 & $\theta_{L L i m},(20)$ & $5^{\circ}$ & $8^{\circ}$ \\
\hline Attractor & -- & 1 & Radii of the three attractor groups $\left(R_{A t t r}{ }^{i}\right),(21)$ & -- & $2000 \mathrm{~m} / 3000 \mathrm{~m} / 4000 \mathrm{~m}$ \\
\hline
\end{tabular}

TABLE 9: Parameters of the violation functions associated with hard criteria/constraints.

\begin{tabular}{|c|c|c|c|c|c|}
\hline \multirow{2}{*}{ Criterion } & \multicolumn{2}{|c|}{ Violation factors $p_{j},(16)$} & \multirow{2}{*}{ Parameter } & \multicolumn{2}{|c|}{ Values } \\
\hline & Scenario 1 & Scenario 2 & & Scenario 1 & Scenario 2 \\
\hline \multirow{3}{*}{ Interference with obstacles } & \multirow{3}{*}{5} & \multirow{3}{*}{1} & Severity values, (22) & & \\
\hline & & & Level 2 (red) & \multicolumn{2}{|c|}{1} \\
\hline & & & Level 3 (black) & \multicolumn{2}{|c|}{100} \\
\hline Self-crossing & 1 & 10 & -- & -- & -- \\
\hline \multirow[t]{2}{*}{ Min. length of straight sections } & 1 & 1 & $L_{\min },(24)$ & \multirow[t]{2}{*}{$100 \mathrm{~m}$} & \multirow{2}{*}{$500 \mathrm{~m}$} \\
\hline & & & $R_{\min },(26)$ & & \\
\hline \multirow{3}{*}{ Min. radius of curvature } & \multirow{3}{*}{1} & \multirow{3}{*}{1} & Friction coefficient $(\mu)$ & & o Table 5 \\
\hline & & & $\begin{array}{l}\text { Residual tension } \\
\left(T_{\text {residual }}\right)\end{array}$ & & \\
\hline & & & Pipe weight $w_{s}$ & Calculated & e properties (Table 4) \\
\hline
\end{tabular}

case studies. Those values were tuned through preliminary studies, considering the characteristics of the respective scenario and the previous experience in the design of submarine pipeline routes.

The objective function of (15) does not incorporate the hard criteria that formally characterize design constraints; as shown in Section 4.2.2, these criteria are evaluated separately by the $\varepsilon$-constrained method, being incorporated into $\phi$ (function of the sum of violations $v_{j}(\mathbf{x}),(16)$ ). Table 9 presents the user-assigned values for the parameters associated with these hard criteria/design constraints, also tuned through preliminary studies considering the previous experience of the designer.

It is interesting to recall that the optimization tool must perform checks for the whole series of nodes and segments, into which each candidate route is discretized: not only the OBS criteria (as mentioned in Section 3.1), but also all other criteria described in Section 4 whose parameters vary along the spatial domain depending on the water depth, seabed bathymetry and obstacles, and the pipe and soil properties as seen in Tables 4 and 5.

5.3. Additional Parameters for the OBS Criteria. Besides the pipe and soil properties, evaluation of the OBS criteria also requires the definition of a set of environmental loading cases. According to [21], two extreme loading combinations of wave and current should be considered: (1) 100-year return wave combined with 10-year current and (2) 10-year wave with 10-year current. Wave and current are taken as aligned along eight directions (N, NE, E, SE, S, SW, W, and NW). Table 10 presents the JONSWAP spectral wave parameters (significant height $H s$, peak period $T p$ ); Table 11 presents the extreme near-bottom current velocities $v_{c}$, taken from metocean data for the two scenarios. Figure 8 presents polar graphs depicting the main current and wave parameters, indicating that the predominant environmental loadings are acting along the NE-SW direction. 
TABLE 10: Wave parameters.

\begin{tabular}{|c|c|c|c|c|}
\hline \multirow{2}{*}{ Direction } & \multicolumn{2}{|c|}{ 10-year } & \multicolumn{2}{|c|}{ 100-year } \\
\hline & $H_{s}(\mathrm{~m})$ & $T_{P}(\mathrm{~s})$ & $H_{s}(\mathrm{~m})$ & $T_{P}(\mathrm{~s})$ \\
\hline $\mathrm{N}$ & 4.74 & 9.2 & 5.01 & 9.56 \\
\hline $\mathrm{NE}$ & 4.88 & 9.47 & 5.17 & 9.7 \\
\hline E & 4.34 & 9.9 & 4.87 & 10.4 \\
\hline SE & 5.72 & 10.28 & 6.53 & 11.63 \\
\hline S & 6.19 & 13.54 & 7.1 & 14.35 \\
\hline SW & 7.16 & 14.78 & 7.84 & 15.55 \\
\hline W & 3.57 & 8.22 & 3.88 & 8.51 \\
\hline NW & 3.57 & 8.22 & 3.88 & 8.51 \\
\hline
\end{tabular}

TABLE 11: Near bottom current velocities (m/s).

\begin{tabular}{|c|c|c|c|c|c|c|c|c|}
\hline \multirow{3}{*}{ Direction } & \multicolumn{2}{|c|}{ All scenarios } & \multicolumn{6}{|c|}{ Scenario 2} \\
\hline & \multicolumn{2}{|c|}{ Current 1} & \multicolumn{2}{|c|}{ Current 2} & \multicolumn{2}{|c|}{ Current 3} & \multicolumn{2}{|c|}{ Current 4} \\
\hline & $10-\mathrm{yr}$ & $100-\mathrm{yr}$ & $10-y r$ & $100-\mathrm{yr}$ & $10-\mathrm{yr}$ & $100-\mathrm{yr}$ & $10-\mathrm{yr}$ & $100-\mathrm{yr}$ \\
\hline $\mathrm{N}$ & 0.54 & 0.65 & 1.45 & 1.92 & 0.57 & 0.71 & 0.36 & 0.45 \\
\hline $\mathrm{NE}$ & 0.75 & 0.96 & 1.13 & 1.43 & 0.52 & 0.63 & 0.44 & 0.56 \\
\hline $\mathrm{E}$ & 0.62 & 0.74 & 1.51 & 2.06 & 0.52 & 0.65 & 0.39 & 0.5 \\
\hline SE & 0.65 & 0.77 & 1.6 & 2.03 & 0.5 & 0.64 & 0.29 & 0.4 \\
\hline S & 0.96 & 1.18 & 1.05 & 1.2 & 0.57 & 0.74 & 0.31 & 0.41 \\
\hline SW & 1.09 & 1.41 & 0.76 & 0.85 & 0.48 & 0.62 & 0.34 & 0.45 \\
\hline W & 0.63 & 0.75 & 0.92 & 1.08 & 0.38 & 0.47 & 0.56 & 0.77 \\
\hline NW & 0.55 & 0.61 & 1.11 & 1.37 & 0.41 & 0.5 & 0.42 & 0.54 \\
\hline
\end{tabular}
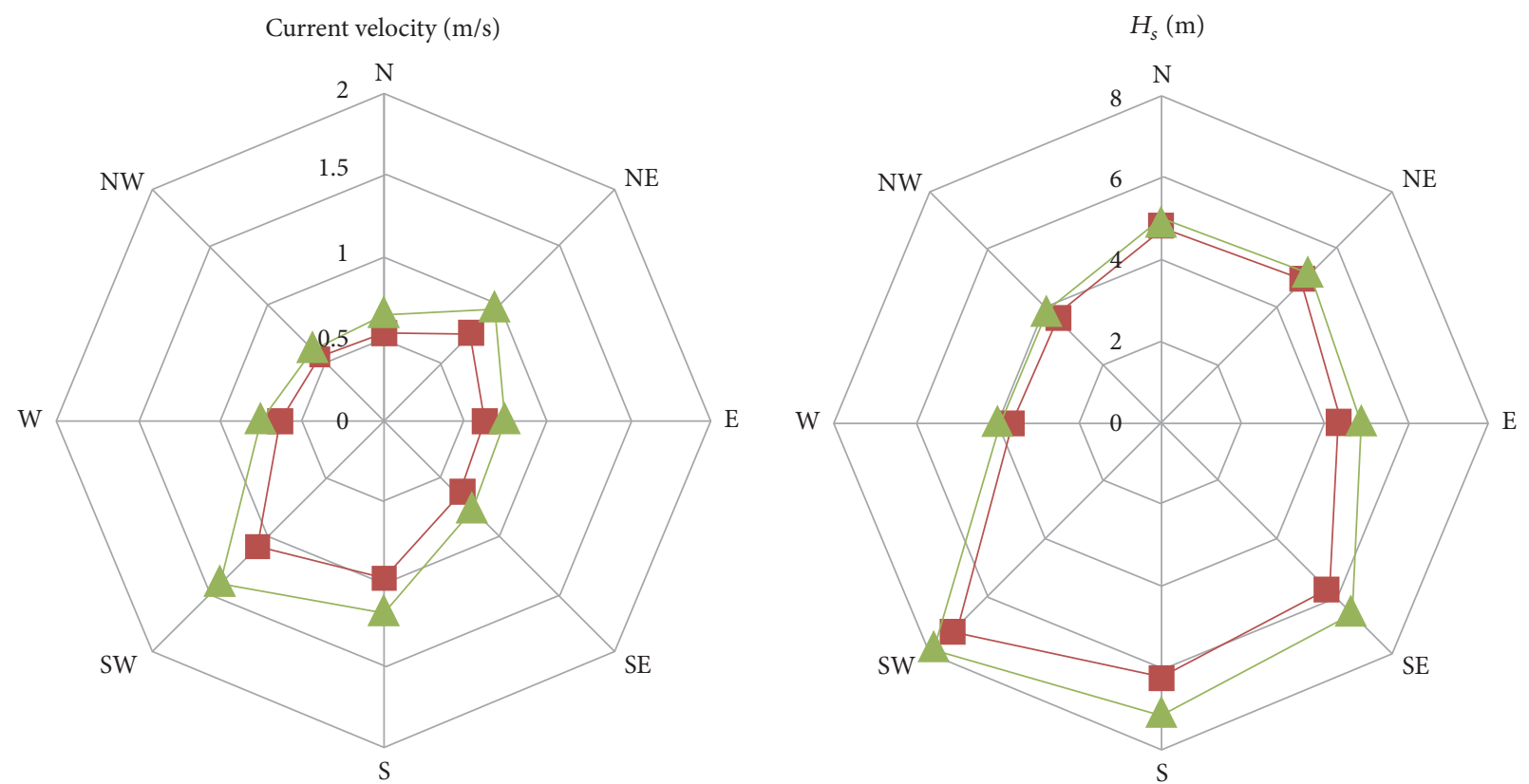

10 years

100 years

10 years

100 years

FIGURE 8: Environmental loadings. 


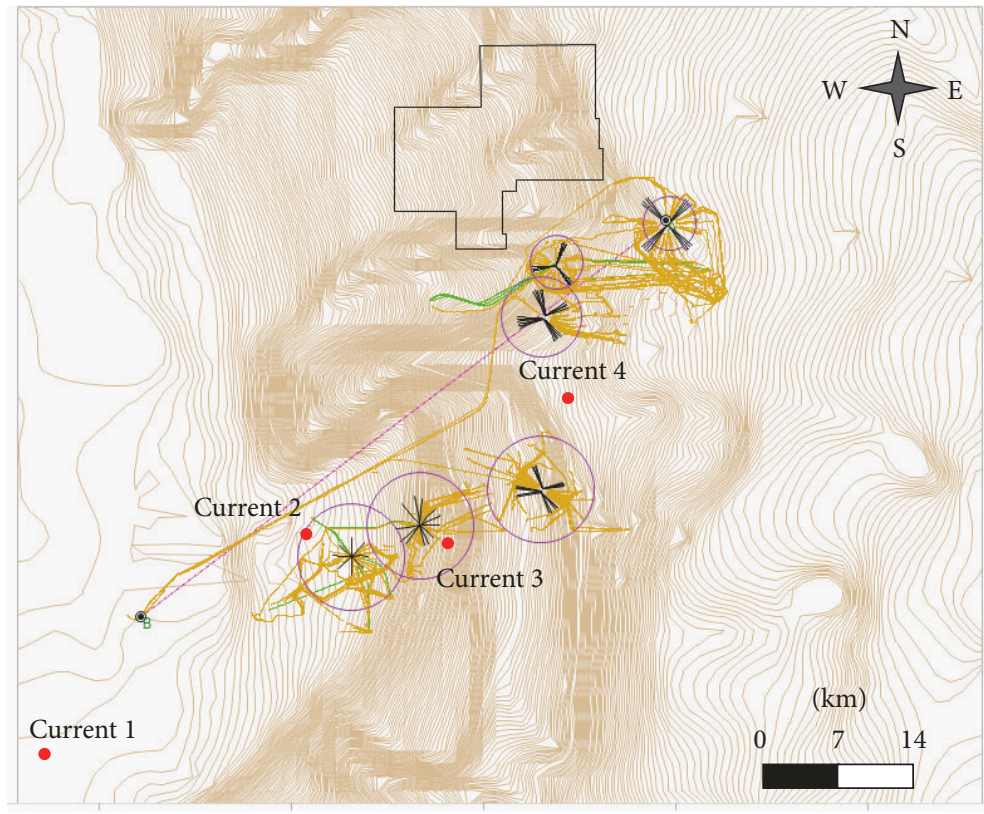

FIGURE 9: Position of the current measurements.

As seen in Table 11 for the more complex scenario 2, the metocean data indicate that the current values vary along the spatial domain according to four points of measurement depicted in Figure 9. Thus, the optimization tool must evaluate the current loading along each node/segment of a route, by taking the values corresponding to the measurement point that is nearest to the node.

The near-bottom current velocities perpendicular to the pipeline are calculated for each node of each candidate route by applying a factor to take into account the directionality and also the effect of the bottom boundary layer. For this purpose the following expression [21] is considered, where the directionality is taken into account by the angle between current direction and axis of the pipe segment $\theta_{c}$; and the bottom boundary layer is a function of the elevation above sea bed $z$, bottom roughness parameter $z_{0}$ (whose values are defined in [21]), and reference measurement height over seabed $z_{r}$ :

$$
V(z)=V\left(z_{r}\right) \cdot \frac{\ln \left(z+z_{0}\right)-\ln \left(z_{0}\right)}{\ln \left(z_{r}+z_{0}\right)-\ln \left(z_{0}\right)} \sin \left(\theta_{c}\right) .
$$

This expression is employed for all directions of current, with their respective values of velocity and relative angle $\theta_{c}$. Taking all resulting perpendicular velocities, the respective ballast weights per unit length for the node/segment are calculated following the procedures described in Section 3.3 that also takes into account the corresponding values of transversal declivity; the required ballast weight is then taken as the higher value amongst the obtained results (eventually, smaller velocity values associated with higher declivities might require more ballast).

The near-bottom wave-induced water velocities perpendicular to the pipeline must also be evaluated for each node, since they depend on the water depth. Moreover, recalling that wave loadings are relevant only for pipelines in shallower waters, the optimization tool automatically disregards wave effects for pipe segments resting in water depths larger than a user-defined limit (for instance, $100 \mathrm{~m}$ ). Thus, as can be seen in Table 3, wave loadings are relevant only for the routes of scenario 1, where a significant part of the pipeline rests on shallow waters.

Moreover, according to [21] the "generalized stability" criterion is more adequate for shallower waters, so a depth limit may also be taken to allow the selection of this criterion. Thus, while both the "absolute" and the "generalized" criteria are employed and compared for the shallower segments of scenario 1 (the latter considering the allowable displacement as 10 times the pipe diameter), only the absolute stability criterion is activated for the deeper segments of scenario 1 and for the routes of scenario 2 .

5.4. Results. The results for the case studies are compared in terms of figures that show the geometry of the optimal route, indicating the ballast weight required for stability by a color gradation scheme (where green, yellow, and red indicate successively higher weight values), and in tables that present the corresponding values for the main parameters of the objective function, for example, length $L_{\text {Route }}$, total ballast weight $W_{b \text {, Route }}$, and ballast weight per unit length $w_{b \text {,pipe }}$, according to (4) and (5).

5.4.1. Scenario 1. For this simpler scenario two sets of optimization runs were performed, employing, respectively, the "favoring length" and "favoring ballast" weighting factors $k_{L}$ and $k_{W b}$ of Table 7 . Each set consists of two runs: the 
TABLE 12: Scenario 1: parameters of the optimal routes.

\begin{tabular}{lcccc}
\hline & \multicolumn{2}{c}{ Absolute stability } & \multicolumn{2}{c}{ Absolute + generalized } \\
& Favoring length & Favoring ballast & Favoring length & Favoring ballast \\
\hline Route length $(\mathrm{m})$ & 12,400 & 15,060 & 12,403 & 4,812 \\
Total ballast weight $(\mathrm{kN})$ & 5,208 & 3,042 & & \\
Ballast weight per unit length $(\mathrm{N} / \mathrm{m})$ & & & 388 & 14,792 \\
Average & 420 & 202 & 100 & 0 \\
Min. & 100 & 0 & 4,500 & 2,647 \\
Max. & 4,500 & 2,900 & 2,500 \\
\hline
\end{tabular}

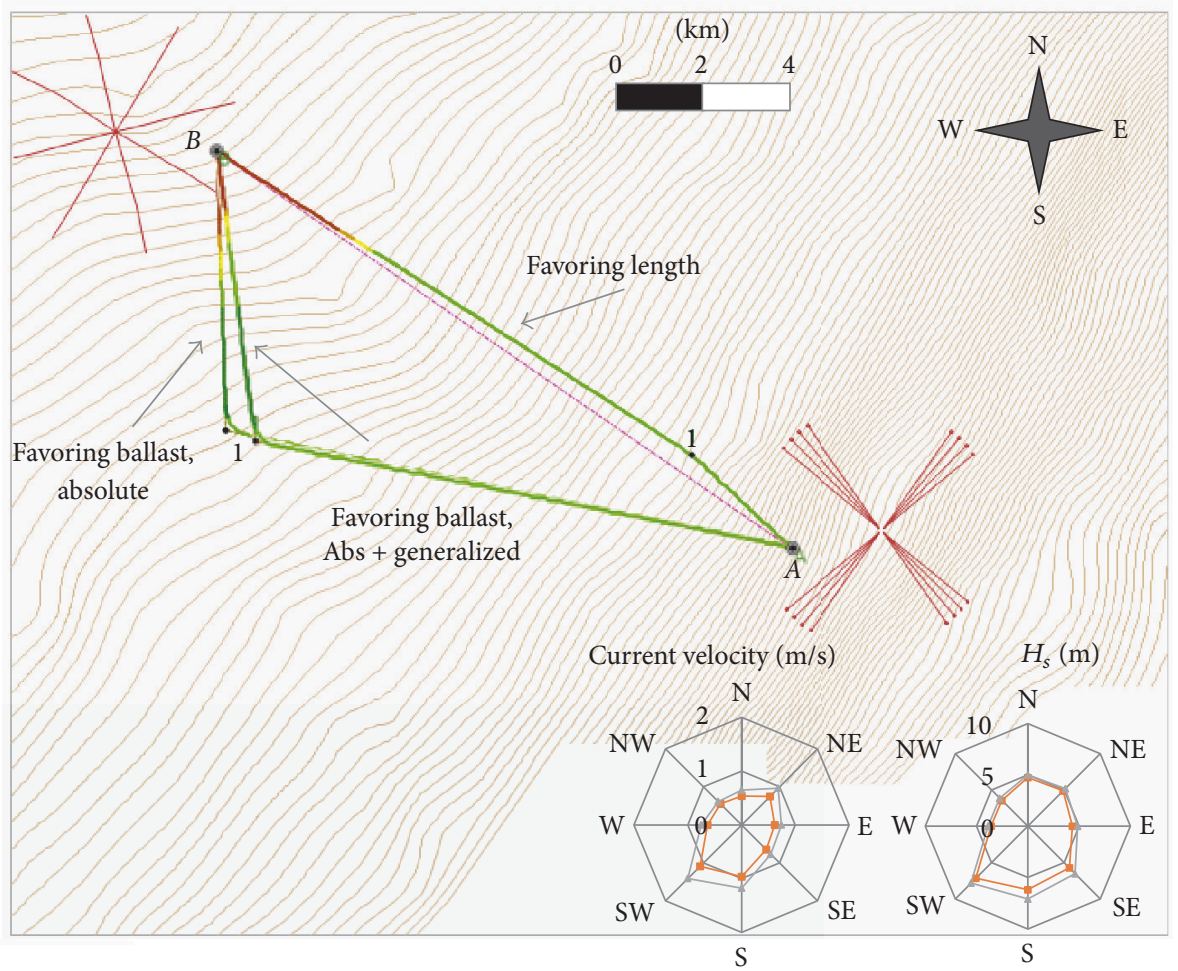

Ballast:

$$
\begin{aligned}
& 0-100 \mathrm{~N} / \mathrm{m} \\
& -100-500 \mathrm{~N} / \mathrm{m} \\
& -500-4500 \mathrm{~N} / \mathrm{m}
\end{aligned}
$$

FIGURE 10: Scenario 1: comparison of optimal routes.

first using only the "absolute stability" OBS criterion and the second set considering both the absolute and generalized criteria, automatically selected according to the water depth.

Figure 10 shows the resulting optimal routes, while Table 12 compares the routes in terms of the main parameters of the objective function, that is, route length and ballast weight. As expected, the optimization runs with the "favoring length" weighting factors $k_{L}$ and $k_{W b}$ of Table 7 generated shorter optimal routes, closer to the straight line between endpoints $A B$; the OBS criteria did not affect their geometries, so they are practically coincident and cannot be distinguished in Figure 10. However, they required considerably more ballast weight, mostly for the pipe segments closer to endpoint $B$ (located in shallower waters), as can be seen from the lengthier segments depicted in red and yellow in Figure 10.

On the other hand, the routes generated with the "favoring ballast" weighting factors have lengths that are not dramatically greater (20\%, approximately), while requiring almost half of the total ballast compared with the "favoring length" routes; in this sense, perhaps this choice of favoring ballast minimization may lead to a more efficient design. This is mostly due to the straight section $\overline{B 1}$ located in shallower depths ranging from 20 to $150 \mathrm{~m}$, where wave loads are more significant; in Figure 10 it can be seen that, to reduce the resultant loads normal to the pipeline axis, the optimization algorithm tries to align the route with the most severe directions of environmental loadings (NE-SW and 


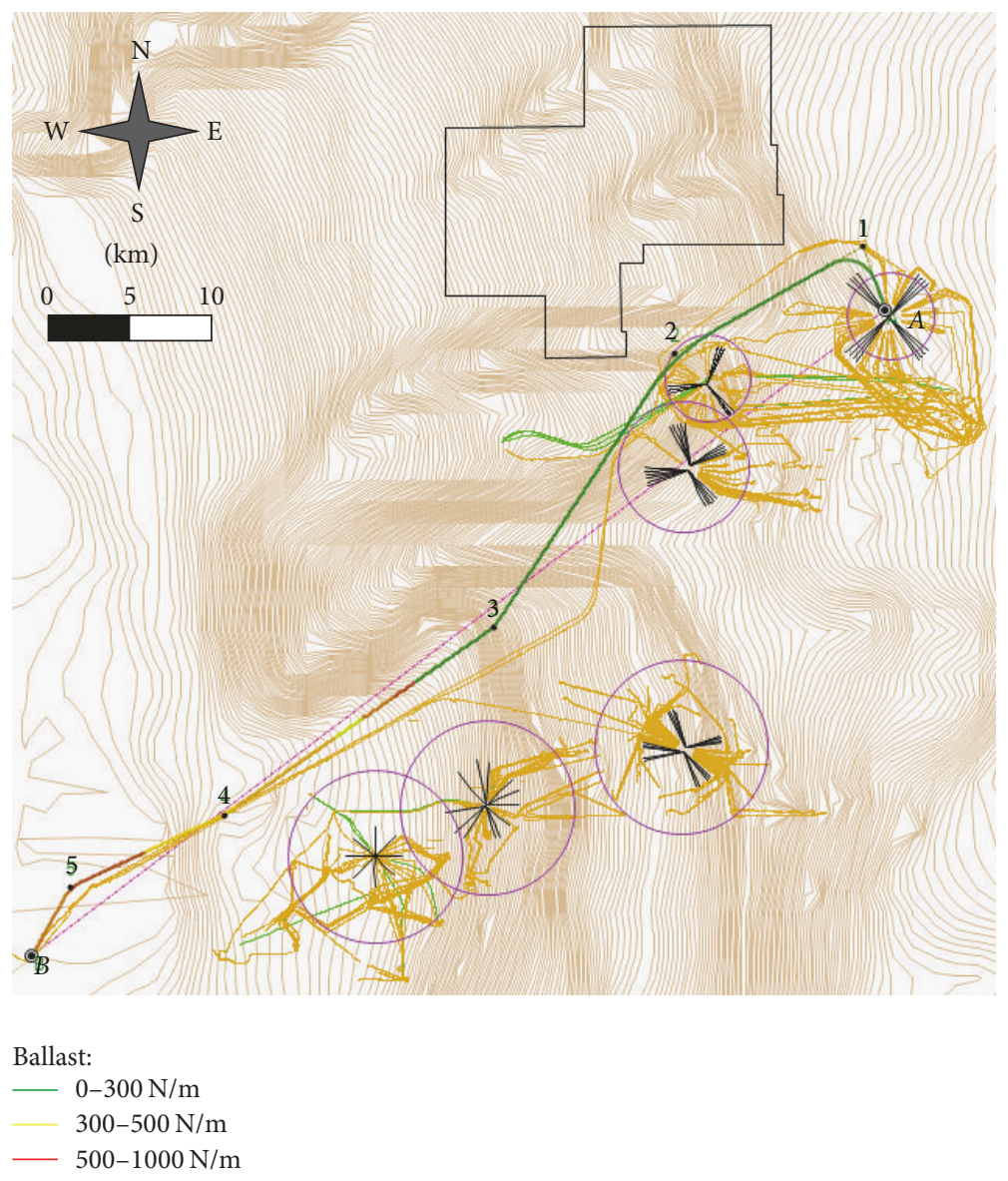

FIGURE 11: Scenario 2: “favoring length" route.

TABLE 13: Scenario 2: parameters of the optimal routes.

\begin{tabular}{lccc}
\hline & Favoring length & Intermediate & Favoring ballast \\
\hline Route length $(\mathrm{m})$ & 53,923 & 55,966 & 56,870 \\
Total ballast weight $(\mathrm{kN})$ & 14,508 & 13,564 & 13,206 \\
Average ballast weight per unit length $(\mathrm{N} / \mathrm{m})$ & 269 & 242 & 232 \\
\hline
\end{tabular}

$\mathrm{N}-\mathrm{S}$ directions), just enough to avoid interference with the mooring line close to endpoint $B$.

Differently from the "favoring length" routes, the "favoring ballast" routes provided by the different stability criteria can be clearly identified in Figure 10 (indicated as "favoring ballast, absolute" and "favoring ballast, Abs + generalized," respectively). Table 12 confirms that the "generalized stability" criterion is indeed less conservative, not only leading to shorter routes, but also requiring less ballast. This latter aspect is also indicated by the shorter length of the segments that require more ballast (depicted in red in Figure 10).

5.4.2. Scenario 2. The optimal routes obtained for scenario 2 , corresponding to the three different sets of values for the weighting factors of Table 7 , are shown next: favoring length (Figure 11), intermediate (Figure 12), and favoring ballast (Figure 13). Figure 14 groups those three routes for a better visual assessment.

Table 13 presents the values for the main parameters of the objective function: length and ballast weight. Again, as expected, increasing the weighting factor $k_{L}$ leads to the reduction of the route length and conversely to the increase in ballast weight. Thus the "favoring length" route requires more ballast, especially in the shallower region near endpoint $B$ and also in the stretch depicted in red between points 3 and 4 indicated in Figure 11. This stretch extends from the depth of $300 \mathrm{~m}$ that sets the limit where the soil changes from sand to clay, thus affecting the OBS calculations; see Table 5 and Figure 15 where sand and clay regions are depicted in gray and green, respectively.

The other routes ("intermediate" and "favoring ballast") require slightly less ballast in this stretch. Overall, the 


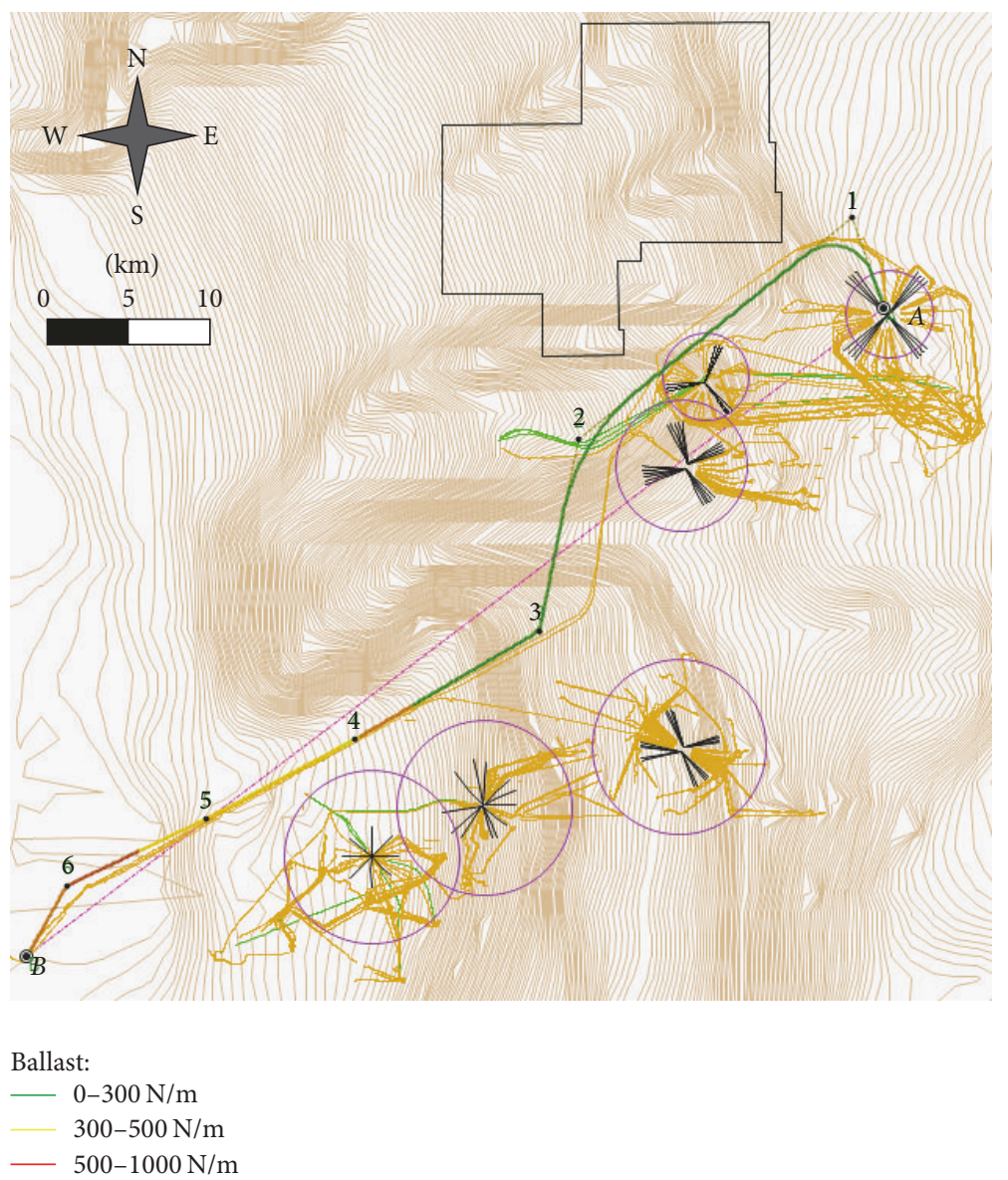

FIGURE 12: Scenario 2: “intermediate" route.

"favoring ballast" route is longer but requires less ballast, while the "intermediate" route is around $900 \mathrm{~m}$ shorter but requires slightly more ballast (4\%). Another advantage of the "favoring ballast" route is that it deviates from the flowlines (depicted in light green, close to point 3, in Figure 13), thus minimizing the requirements for intervention to avoid interference with obstacles.

In summary, for this considerably complex scenario with a greater distance between the endpoints (approx. $50 \mathrm{~km}$ ) and irregular seabed bathymetry (including canyons), it can be seen that the optimal routes successfully complied with the main objectives and constraints of the optimization procedure. Excessive declivities associated with the crossing of canyons have been minimized; the obstacle indicated by a black polygon (representing a hard constraint) has been avoided; and the proximity to the other platforms (corresponding to the "attractors" depicted as purple circles) has been attained as close as possible.

\section{Final Remarks and Conclusions}

It is well known that the costs of a given pipeline project are dictated not only by the material of the pipe segments themselves (that derives from the route length), but also by installation and intervention procedures that mitigate problems related to the structural behavior of the pipe under hydrostatic and environmental loadings. Considering specifically on-bottom stability, several methods to provide ballast and stabilize the pipe (including those described at the end of Section 3.3) introduce substantial costs to the installation of the pipeline along the chosen route. In the traditional pipeline design methodology, the definition of a route and the stability calculations had been performed independently: firstly, the route is defined according to geographical-topographical issues, including seabed bathymetry and obstacles; then stability is verified and the required mitigating procedures are specified.

Now, with the incorporation of OBS criteria into the route optimization tool following the approaches described in this work, stability becomes an integral part of the route optimization process. This may be seen as a breakthrough in the traditional design methodology, allowing the designer to simultaneously handle the minimization of route length and the cost of the mitigating procedures.

The optimization tool provides the decision-maker with profitable information regarding the optimal routes, considering different aspects of the design and the costs associated with the different procedures that provide ballast to stabilize 


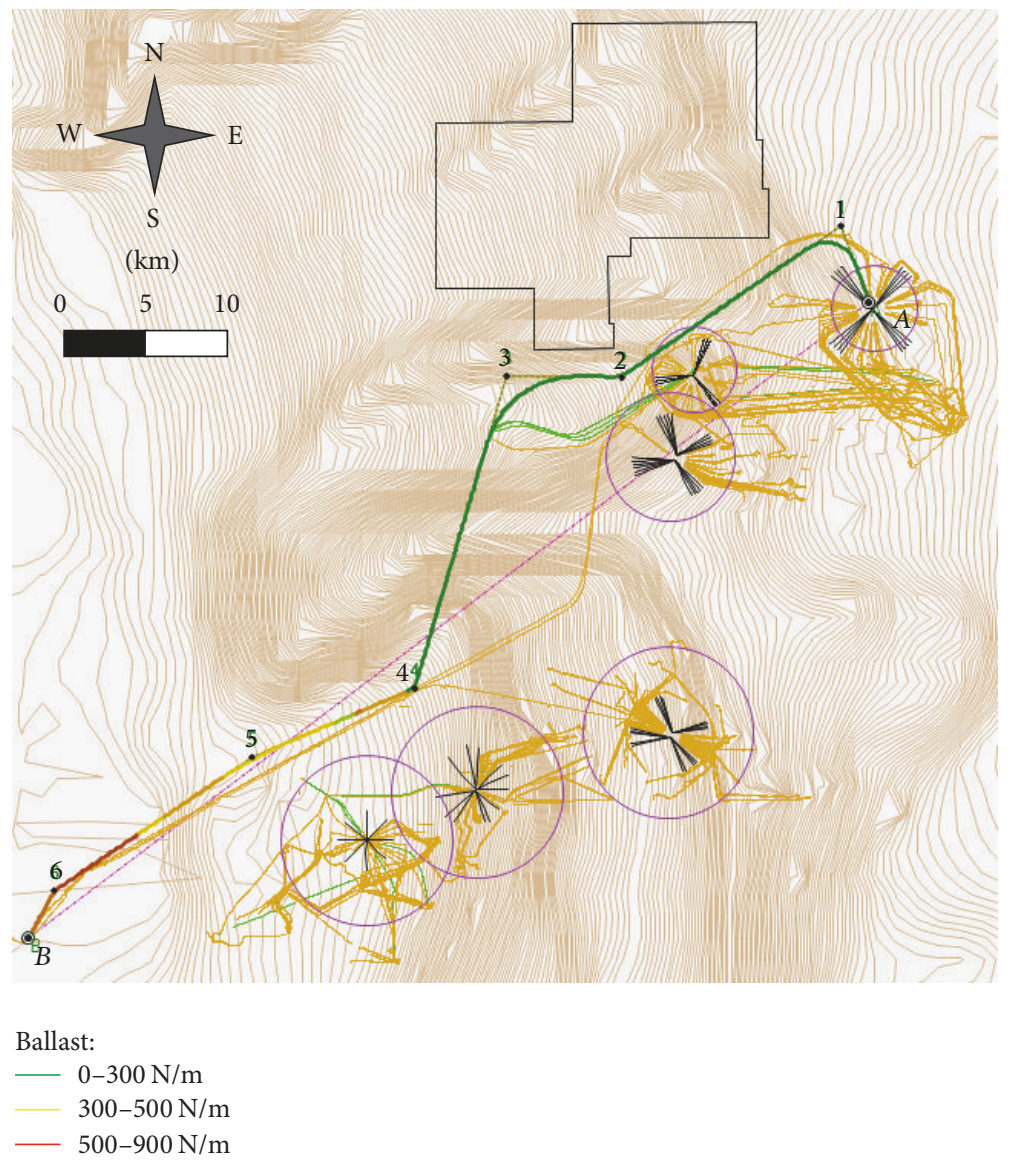

FIGURE 13: Scenario 2: "favoring ballast" route.

a pipeline (mattress, trenching, burying, concrete coating, or even employing a heavier pipe with additional steel wall thickness). For instance, relatively high values for the weight of concrete might hinder the installation of the pipeline (due to limitations of the launching vessel and/or excessive stresses on the pipe itself). In such cases, the designer might then opt for a hybrid solution combining less concrete coating with a heavier pipe (with increased wall thickness). Taking the required ballast weights as indicated in Table 12 or Table 13, different combinations of steel wall and concrete coating thickness may be provided by the iterative procedure described at the end of Section 3.3.

Overall, the computational costs associated with the stability calculations by the different OBS methods ("absolute stability" and "generalized stability") are relatively low, corroborating the feasibility of their incorporation into the optimization tool. The results of the first case study confirmed that the latter is more adequate for shallower waters with increased influence of the environmental loads on the pipeline: it leads to smaller routes and/or less ballast when compared with those obtained by the absolute method. Thus, also observing that the computational costs associated with the "generalized" criterion are not significantly higher than those for the "absolute" criterion, in the optimization tool the former may be set as the default procedure for pipe segments below a given user-specified water depth value.

Several new developments related to the route optimization tool are currently underway, including procedures to incorporate other technical/engineering criteria into the objective function (for instance, VIV-induced fatigue on free spans and multiphase flow). Also, here the multiobjective problem of optimizing the route length and ballast weight has been dealt with by combining the respective terms into a single objective function. As observed in the results of the case studies, those may be conflicting objectives since usually the optimal routes with shorter lengths have more ballast weight and vice versa. While the present approach effectively provides different optimal routes by adjusting the weighting factors $k_{L}$ and $k_{W b}$ along different optimization runs, another promising approach would be to formally model the optimization problem with a multiobjective procedure using specialized algorithms based on the Pareto front concept, thus obtaining a set of optimal routes from a single optimization run. Preliminary studies related to those topics have been briefly outlined in conference papers [24, 25]; more detailed and conclusive results will be presented in subsequent works. 


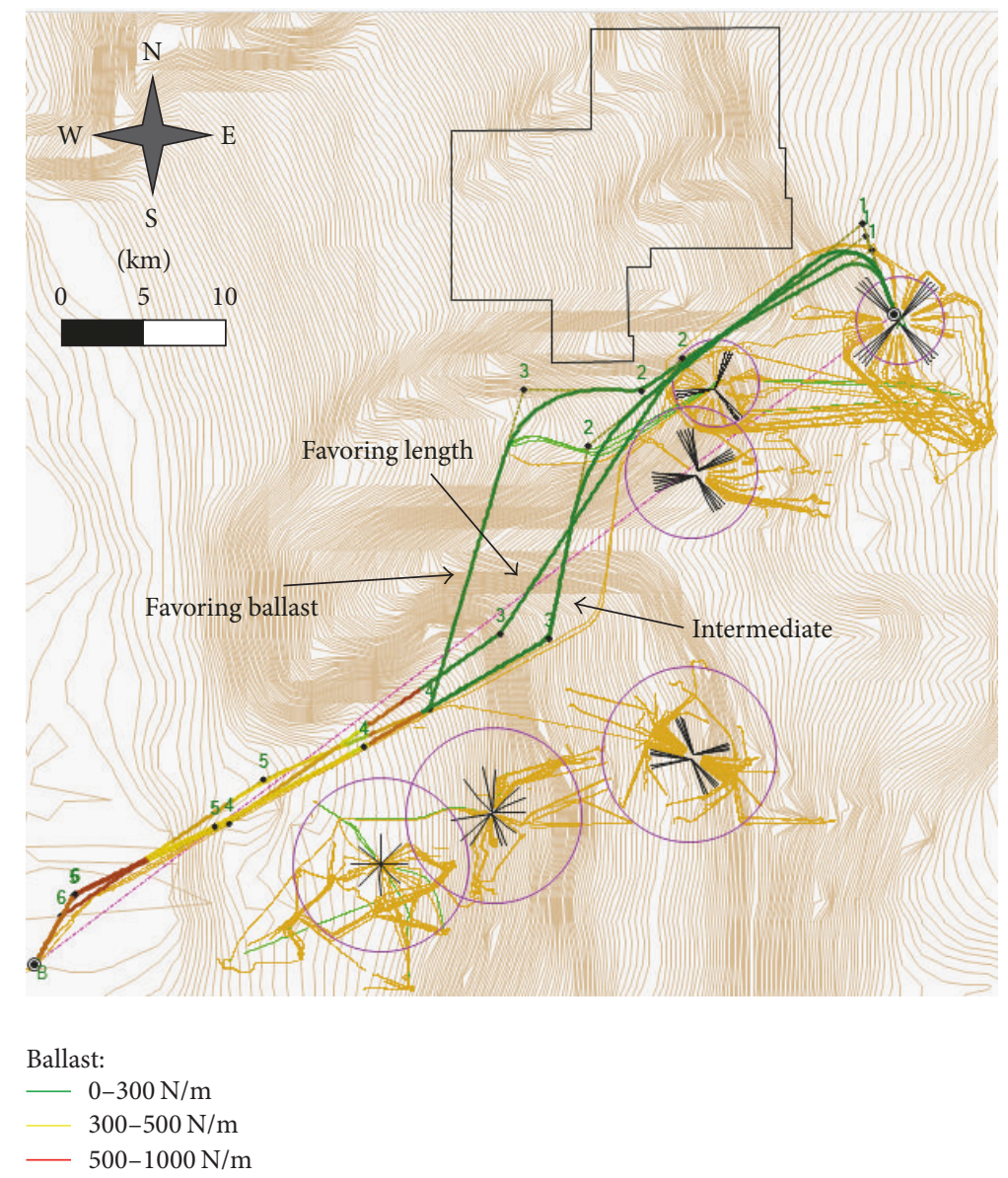

FIGURE 14: Scenario 2: comparison of optimal routes.

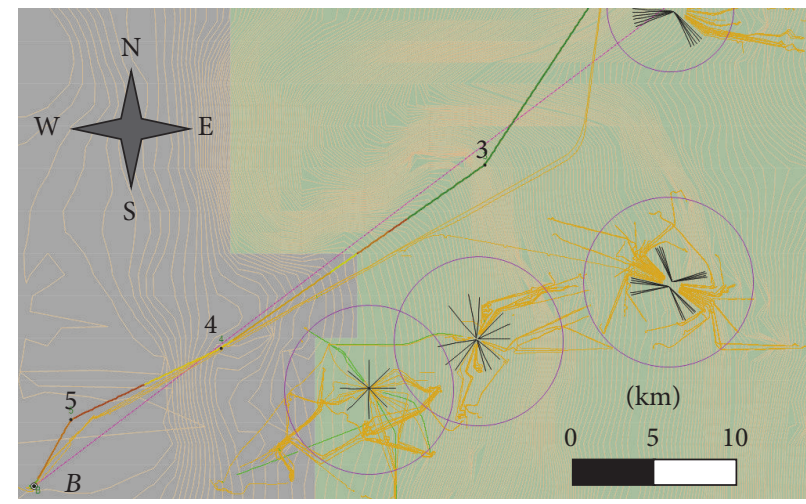

FIgURE 15: Scenario 2: "favoring length" route, detail.

To summarize, the main feature of the developments presented in this work consists of anticipating, to the stage of route definition, engineering checks and calculations traditionally related to subsequent design stages. Thus the optimization tool may reduce the design time of a given pipeline, not only for the route definition, but also for the other stages involving the detailed verification of those engineering criteria; more important, the tool can minimize installation and operational costs of the pipeline.

\section{Conflicts of Interest}

The authors declare that there are no conflicts of interest regarding the publication of this article.

\section{Acknowledgments}

The first and second authors would like to acknowledge the Brazilian research funding agencies $\mathrm{CNPq}$ (Conselho 
Nacional de Desenvolvimento Científico e Tecnológico) and FAPERJ (Fundação Carlos Chagas Filho de Amparo à Pesquisa do Estado do Rio de Janeiro) for the financial support of their doctoral studies at PEC/COPPE/UFRJ. The fourth and fifth authors acknowledge the support of $\mathrm{CNPq}$ and FAPERJ corresponding to the research Grants nos. 304850/2012-8, 306104/2013-0, and E-26/201.184/2014, respectively. Finally, all authors acknowledge Petrobras S.A. for supporting and allowing the publication of this work.

\section{References}

[1] Det Norske Veritas, Offshore Standard DNV-OS-F101 Submarine Pipeline Systems, Det Norske Veritas, Oslo, Norway, 2012.

[2] https://stoprust.com/products-and-services/retromat/.

[3] http://allseas.com/activities/pipelines-and-subsea/pipeline-protection/.

[4] http://offshore-fleet.com/data/rock-dumping-vessel.htm.

[5] W. T. Jones, "On-Bottom Pipeline Stability in Steady Water Currents," Journal of Petroleum Technology, vol. 30, no. 3, pp. 475484, 1978.

[6] H. Meisingset, J. Hove, and G. Olsen, "Olsen Optimization of Pipeline Routes," in in the Proceedings of the Fourteenth International Offshore and Polar Engineering Conference, Toulon, France, 2004.

[7] D. H. Fernandes, B. P. Jacob, B. S. L. P. de Lima, and C. H. Albrecht, "Offshore Pipeline Route Assessment via Evolutionary Algorithms," in in the Proceedings of the XXIX Iberian-LatinAmerican Congress on Computational Methods in Engineering, CILAMCE, Maceió, AL, Brazil, 2008.

[8] D. H. Fernandes, B. P. Jacob, B. S. L. P. de Lima, A. R. Medeiros, and C. H. Albrecht, "A Proposal of Multi-Objective Function for Submarine Rigid Pipelines Route Optimization via Evolutionary Algorithms," in in the processdings of Rio Pipeline Conference \& Exposition 2009 Annals, Rio de Janeiro, Brazil, 2009.

[9] S. Percival, D. Hendrix, and F. Noblesse, "Hydrodynamic optimization of ship hull forms," Applied Ocean Research, vol. 23, no. 6, pp. 337-355, 2001.

[10] B. D. S. L. P. de lima, B. P. Jacob, and N. F. F. Ebecken, "A hybrid fuzzy/genetic algorithm for the design of offshore oil production risers," International Journal for Numerical Methods in Engineering, vol. 64, no. 11, pp. 1459-1482, 2005.

[11] R. L. Tanaka and C. D. A. Martins, "Parallel dynamic optimization of steel risers," Journal of Offshore Mechanics and Arctic Engineering, vol. 133, no. 1, Article ID 011302, 2010.

[12] A. A. de Pina, C. H. Albrecht, B. S. de Lima, and B. P. Jacob, "Tailoring the particle swarm optimization algorithm for the design of offshore oil production risers," Optimization and Engineering, vol. 12, no. 1-2, pp. 215-235, 2011.

[13] I. N. Vieira, B. S. de Lima, and B. P. Jacob, "Bio-inspired algorithms for the optimization of offshore oil production systems," International Journal for Numerical Methods in Engineering, vol. 91, no. 10, pp. 1023-1044, 2012.

[14] M. A. L. Martins, E. N. Lages, and E. S. S. Silveira, "Compliant vertical access riser assessment: DOE analysis and dynamic response optimization," Applied Ocean Research, vol. 41, pp. 2840, 2013.

[15] I. N. Vieira, C. H. Albrecht, B. S. L. P. de Lima, B. P. Jacob, D. M. Rocha, and C. O. Cardoso, "Towards a Computational Tool for the Synthesis and Optimization of Submarine Pipeline Routes," in in the proceedings of the Twentieth International Offshore and Polar Engineering Conference - ISOPE, Beijing, China, 2010.

[16] R. R. De Lucena, J. S. Baioco, B. S. L. P. De Lima, C. H. Albrecht, and B. P. Jacob, "Optimal design of submarine pipeline routes by genetic algorithm with different constraint handling techniques," Advances in Engineering Software, vol. 76, pp. 110124, 2014.

[17] D. E. Goldberg, Genetic Algorithms in Search, Optimization and Machine Learning, Addison-Wesley, Boston, Massachusetts, USA, 1989.

[18] K. F. Man, K. S. Tang, and S. Kwong, "Genetic Algorithms: Concepts and Applications," IEEE Transactions on Industrial Eletronics, vol. 43, no. 5, pp. 519-534, 1996.

[19] E. Mezura-Montes and C. A. Coello Coello, "Constraint-handling in nature-inspired numerical optimization: past, present and future," Swarm and Evolutionary Computation, vol. 1, no. 4, pp. 173-194, 2011.

[20] T. Takahama, S. Sakai, and N. Iwane, "Constrained optimization by the epsilon constrained hybrid algorithm of particle swarm optimization and genetic algorithm," in AI 2005: Advances in Artificial Intelligence, Lecture Notes in Artificial Intelligence, pp. 389-400, Springer-Verlag, Berlin, Germany, 2005.

[21] Det Norske Veritas, On Bottom Stability Design of Submarine Pipelines, Recommended Practice DNV-RP-F109, Det Norske Veritas, Oslo, Norway, 2008.

[22] M. H. A. De Lima Jr., J. S. Baioco, C. H. Albrecht et al., "Synthesis and optimization of submarine pipeline routes considering OnBottom Stability criteria," in Proceedings of the ASME 2011 30th International Conference on Ocean, Offshore and Arctic Engineering, OMAE2011, pp. 307-318, Netherlands, June 2011.

[23] B. Guo, S. Song, J. Chacko, and A. Ghalambor, Offshore Pipelines, Gulf Professional Publishing, Elsevier, Houston, Texas, USA, 2005.

[24] J. S. Baioco, P. Stape, M. Granja, C. H. Albrecht, B. S. L. P. De Lima, and B. P. Jacob, "Incorporating engineering criteria to the synthesis and optimization of submarine pipeline routes: Onbottom stability, viv-induced fatigue and multiphase flow," in Proceedings of the ASME 2014 33rd International Conference on Ocean, Offshore and Arctic Engineering, OMAE, San Francisco, California, USA, June 2014.

[25] J. S. Baioco, C. H. Albrecht, B. P. Jacob, and D. M. Rocha, "MultiObjective Optimization of Submarine Pipeline Routes Considering On-Bottom Stability, VIV-Induced Fatigue and Multiphase Flow," in Proceedings of the Twenty-Fifth International Ocean and Polar Engineering Conference - ISOPE, Kona, Hawaii, USA, 2015 


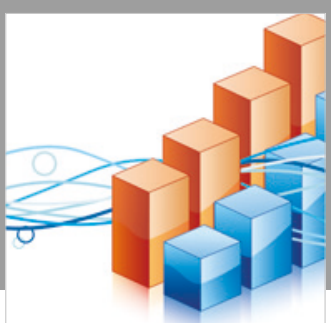

Advances in

Operations Research

\section{-n-m}
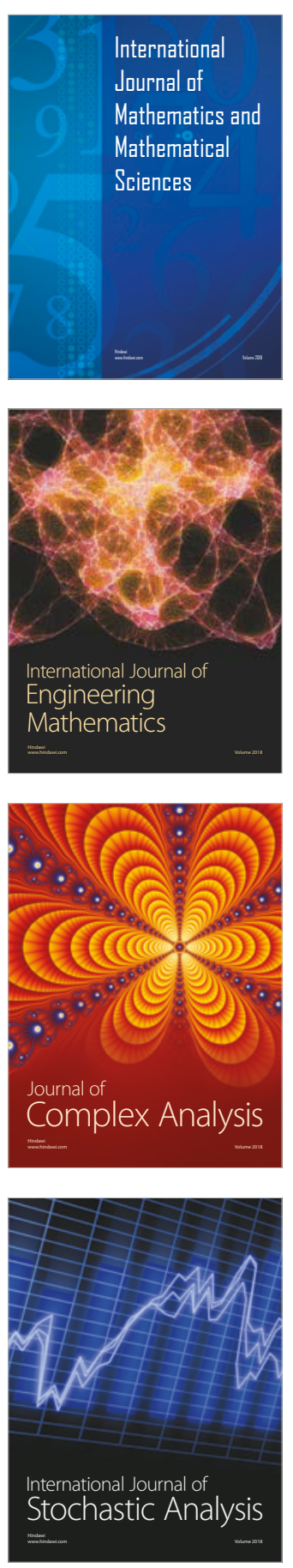
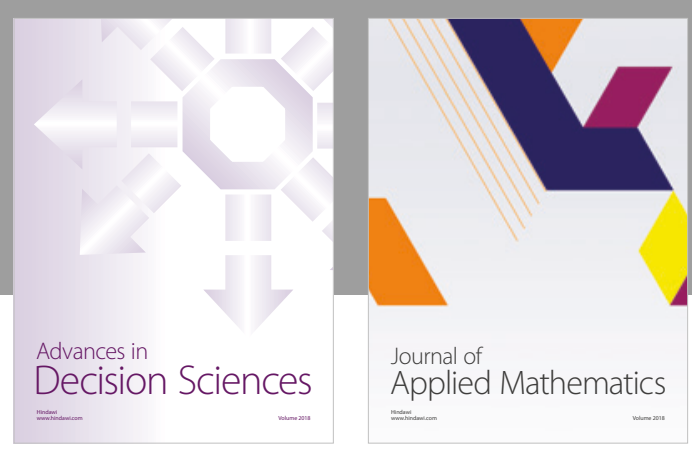

Journal of

Applied Mathematics
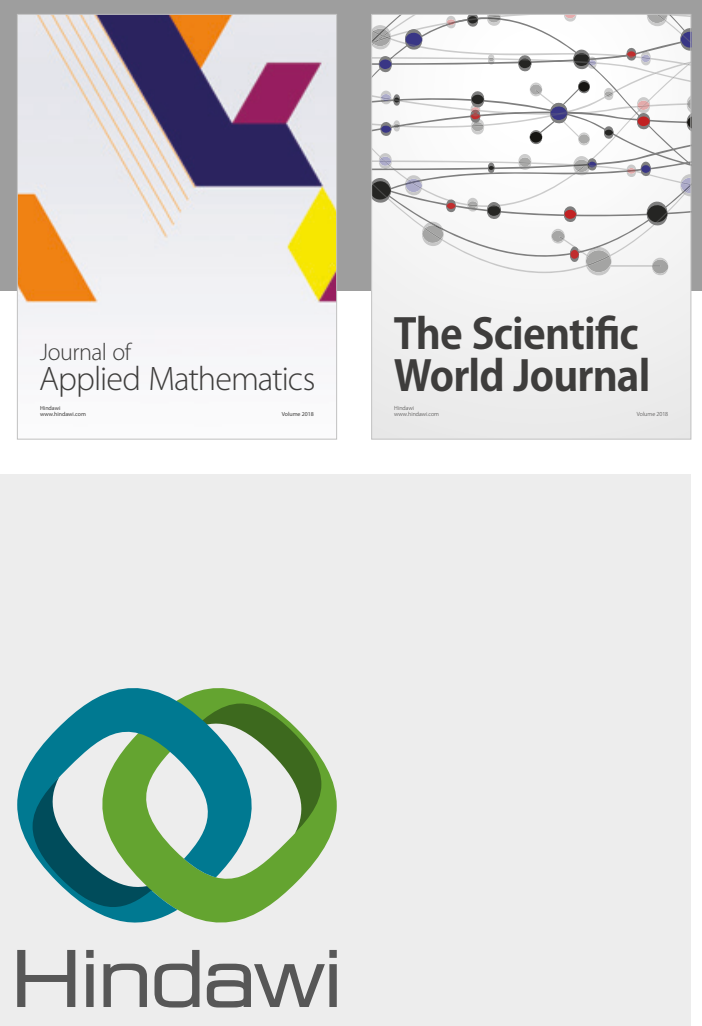

Submit your manuscripts at

www.hindawi.com

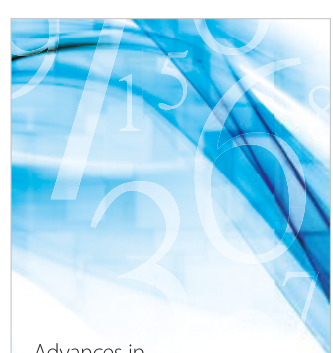

Advances in
Numerical Analysis
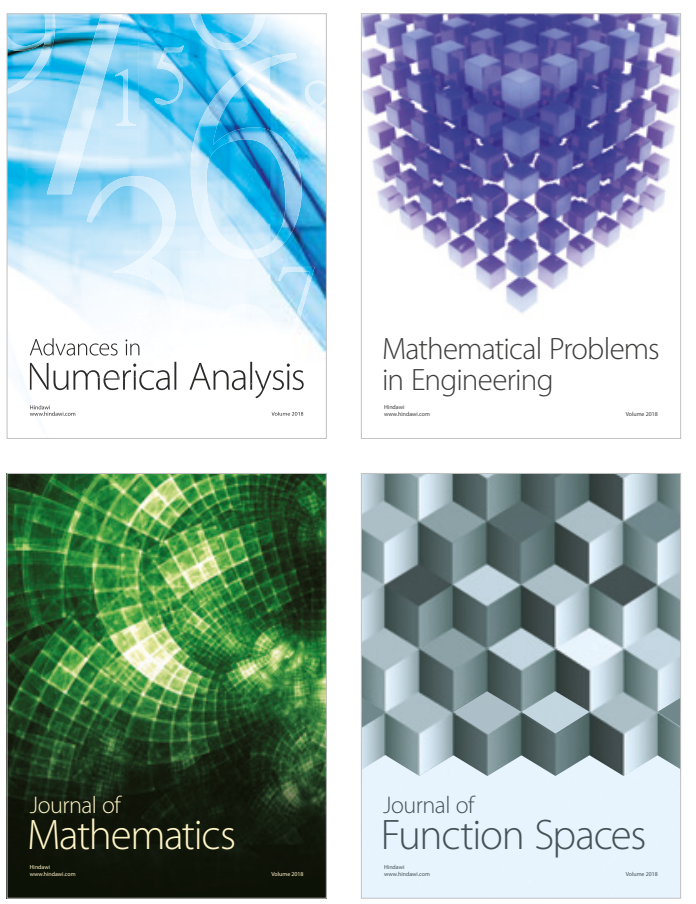

Mathematical Problems in Engineering

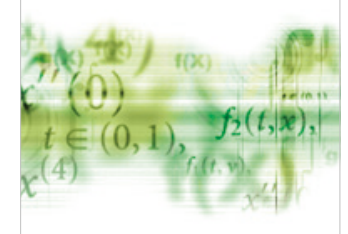

International Journal of

Differential Equations

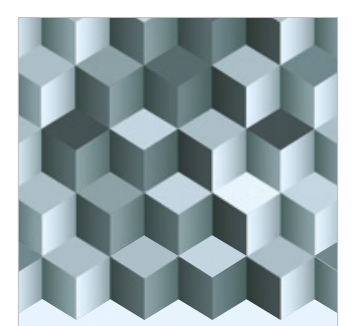

Journal of

Function Spaces

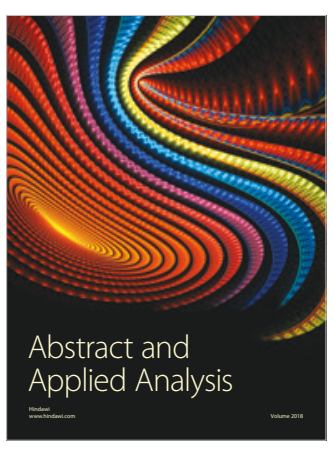

The Scientific

World Journal

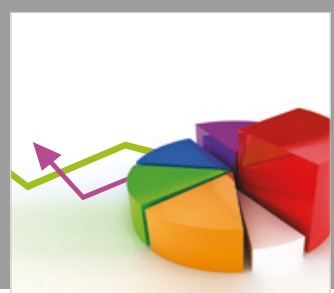

Journal of

Probability and Statistics
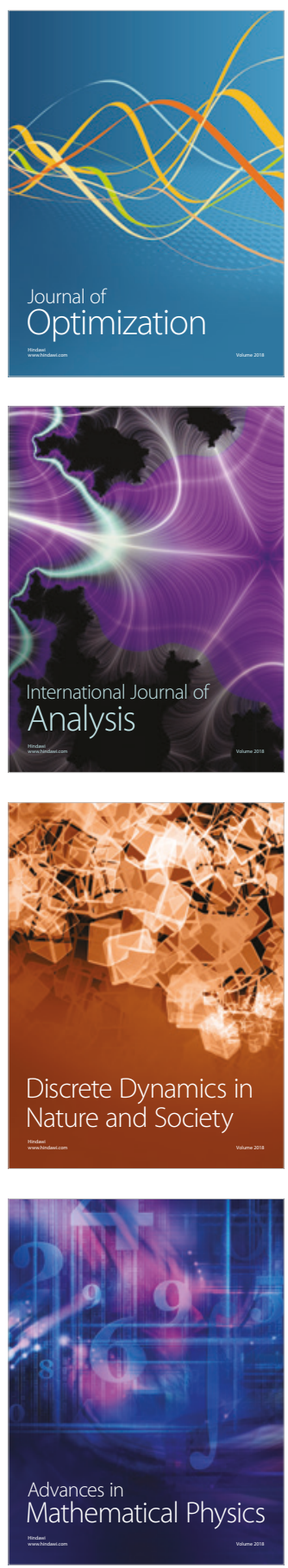